\title{
Multimodal silica nanoparticles are effective cancer-targeted probes in a model of human melanoma
}

\author{
Miriam Benezra, ${ }^{1}$ Oula Penate-Medina, ${ }^{1}$ Pat B. Zanzonico, ${ }^{2}$ David Schaer, ${ }^{3}$ Hooisweng Ow, ${ }^{4}$ \\ Andrew Burns, ${ }^{5}$ Elisa DeStanchina, 6 Valerie Longo, ${ }^{1}$ Erik Herz, ${ }^{7}$ Srikant lyer, ${ }^{7}$ Jedd Wolchok, ${ }^{3,8}$ \\ Steven M. Larson, ${ }^{1}$ Ulrich Wiesner, ${ }^{7}$ and Michelle S. Bradbury ${ }^{1}$

\begin{abstract}
1Department of Radiology, ${ }^{2}$ Department of Medical Physics, and ${ }^{3}$ Immunology Program, Sloan-Kettering Institute for Cancer Research, New York, New York, USA. ${ }^{4}$ Hybrid Silica Technologies, Ithaca, New York, USA. ${ }^{5}$ GE Global Research Center, Niskayuna, New York, USA. ${ }^{6}$ Antitumor Assessment Facility, Sloan-Kettering Institute for Cancer Research, New York, New York, USA.

${ }^{7}$ Department of Materials Science and Engineering, Cornell University, Ithaca, New York, USA.

${ }^{8}$ Department of Medicine, Sloan-Kettering Institute for Cancer Research, New York, New York, USA.
\end{abstract}

\begin{abstract}
Nanoparticle-based materials, such as drug delivery vehicles and diagnostic probes, currently under evaluation in oncology clinical trials are largely not tumor selective. To be clinically successful, the next generation of nanoparticle agents should be tumor selective, nontoxic, and exhibit favorable targeting and clearance profiles. Developing probes meeting these criteria is challenging, requiring comprehensive in vivo evaluations. Here, we describe our full characterization of an approximately 7-nm diameter multimodal silica nanoparticle, exhibiting what we believe to be a unique combination of structural, optical, and biological properties. This ultrasmall cancer-selective silica particle was recently approved for a first-in-human clinical trial. Optimized for efficient renal clearance, it concurrently achieved specific tumor targeting. Dye-encapsulating particles, surface functionalized with cyclic arginine-glycine-aspartic acid peptide ligands and radioiodine, exhibited high-affinity/avidity binding, favorable tumor-to-blood residence time ratios, and enhanced tumor-selective accumulation in $\alpha_{v} \beta_{3}$ integrin-expressing melanoma xenografts in mice. Further, the sensitive, real-time detection and imaging of lymphatic drainage patterns, particle clearance rates, nodal metastases, and differential tumor burden in a large-animal model of melanoma highlighted the distinct potential advantage of this multimodal platform for staging metastatic disease in the clinical setting.
\end{abstract}

\section{Introduction}

Despite recent advances in nanoparticle probe development for biomedicine, the translation of targeted diagnostic particle platforms remains challenging (1-3). Nanoparticle-based materials currently under evaluation in oncology clinical trials are largely nontargeted drug delivery vehicles or devices for thermally treating tissue and not typically surface modified for direct detection by clinical imaging tools. Tumor-selective diagnostic probes, in addition to satisfying critical safety benchmarks, need to elucidate targeted interactions with the microenvironment and their effects on biological systems. Several key factors that limit the translation of such particle probes have been identified: suboptimum pharmacokinetics, in vivo probe nanotoxicity (4), evolving regulatory considerations, reimbursement issues, and resource-intensive scale-up of manufacturing for clinical trials. To address such issues and comply with regulatory and clinical practice guidelines, rigorous quantitative imaging approaches and analysis tools, such as PET, will be essential for evaluating a variety of probes undergoing preclinical testing or transitioning into early-phase clinical trials. By attaching a radiolabel to the particle surface, the clinical potential of these molecularly targeted probes can be sensitively

Authorship note: Miriam Benezra and Oula Penate-Medina contributed equally to this work.

Conflict of interest: Hooisweng Ow and Ulrich Wiesner hold shares of the start-up company Hybrid Silica Technologies.

Citation for this article: J Clin Invest. 2011;121(7):2768-2780. doi:10.1172/JCI45600. and comprehensively defined according to the following set of diagnostic criteria: favorable distribution and targeting kinetics (1, 5-9), efficient renal clearance $(10,11)$, extended circulation (blood residence) times $(5,12,13)$, pronounced tissue signal amplification $(5,6,14)$, and improved tumoral penetration (15).

Recently reported design criteria have been proposed for advancing targeted probes to the clinic (11). These have largely been restricted to improving renal clearance properties of inorganic particles containing inherently toxic components. The relative lack of in vivo toxicology data associated with these probes has led to persistent and valid safety concerns (16), prompting a number of particle-design modifications (9, 17-19), including size, composition, and surface chemistry, that promote more rapid clearance through kidney (renal) or liver (hepatic) pathways (20). While such approaches may minimize toxicity, the ultimate fate and impact of residual particles in the reticuloendothelial system (RES; liver, spleen, lymph nodes, bone marrow) remains unclear, particularly if toxicity is related to prolonged organ residence times $(16,17)$. Accelerated particle clearance may also preclude certain clinical applications as a result of restrictions imposed upon the available imaging period. Further, while rapid particle extravasation may facilitate clearance and delivery to tumors, reduced receptor binding (5) and increased nonspecific tissue dispersal $(21,22)$ may also occur, increasing image background and, potentially, toxicity by prolonging retention.

Only a relatively small percentage of new imaging agents undergoing comprehensive preclinical testing potentially satisfy diagnostic criteria for translation (2). Although a number of fluo- 

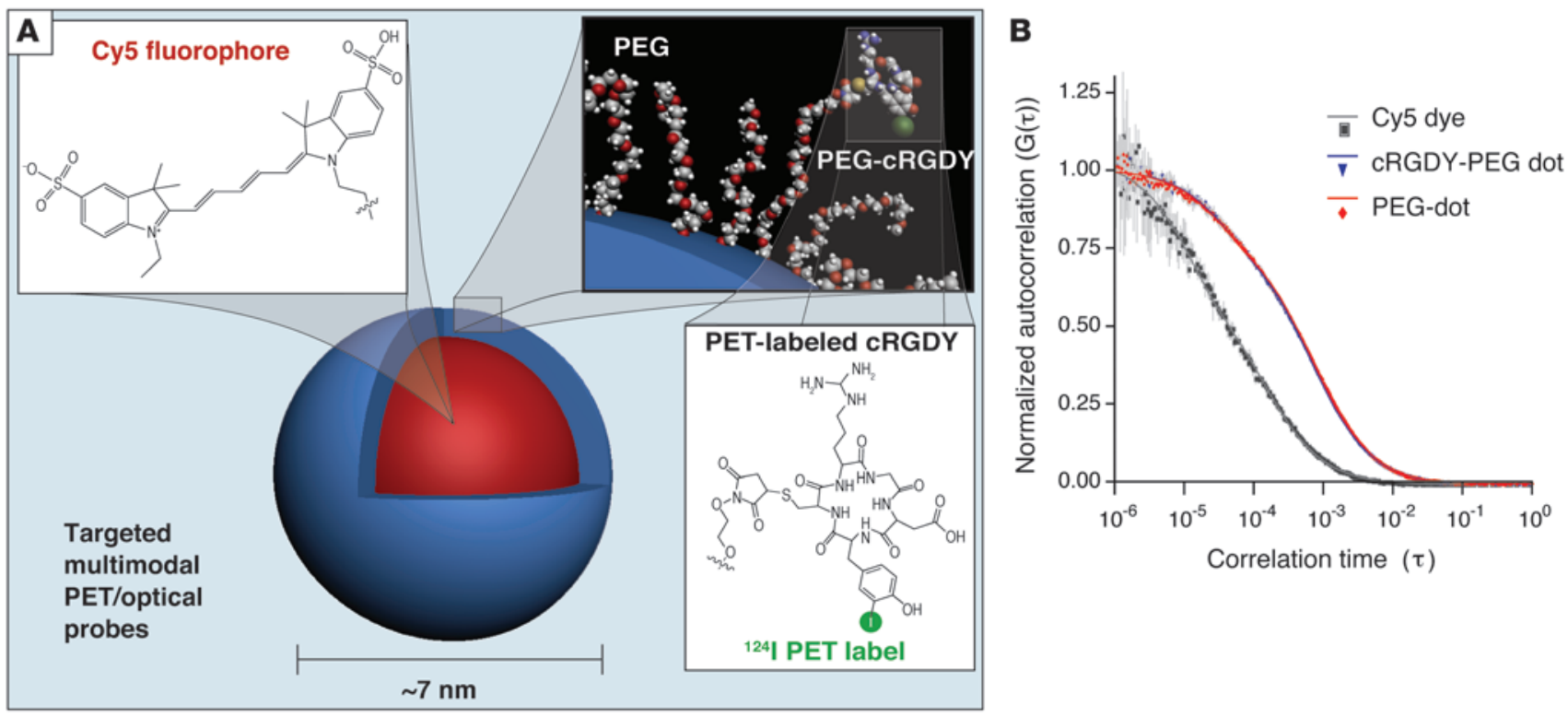

Figure 1

Multimodal $C$ dot design for $\alpha_{v} \beta_{3}$ integrin targeting and characterization. (A) Schematic representation of the ${ }^{124}$ I-cRGDY-PEG-ylated core-shell silica nanoparticle with surface-bearing radiolabels and peptides and core-containing reactive dye molecules (insets). (B) FCS results and single exponential fits for measurements of Cy5 dyes in solution (black) and PEG-coated (PEG-dot, red) and PEG-coated, cRGDY-labeled dots (blue, underneath red data set) showing diffusion time differences as a result of varying hydrodynamic sizes.

rescent particle platforms have been investigated (23-26), only Cornell dots (C dots) meet the aforementioned criteria and have received the first FDA-approved investigational new drug approval of their class and properties for a first-in-human clinical trial. Surface functionalized with small numbers of targeting peptides (cyclic arginine-glycine-aspartic acid [cRGDY] peptides) and longlived radiolabels, this $\alpha_{v} \beta_{3}$ integrin-targeting particle tracer has a unique combination of structural, optical, and biological properties: bulk renal clearance (10), favorable targeting kinetics, lack of acute toxicity, superior photophysical features, and multimodal (PET-optical) imaging capabilities. To the best of our knowledge, these properties have not been collectively achieved or described for any single inorganic particle platform. Importantly, the translation of this cancer-targeted, PET-optical platform represents a significant clinical advance over our previous preclinical optical imaging work using approximately $30-\mathrm{nm}$ in diameter [i.d.] nonPEGylated $(24,27)$ and approximately 3.3 - and 6-nm i.d. PEGylated, nontargeting probes (10) in non-tumor-bearing animals.

We highlight the distinct advantages of using this renally excreted, multimodal agent for tumor-selective targeting and nodal mapping studies in both small- (mouse) and large-animal (miniswine) melanoma models. By combining key benefits of PET (depth penetration, quantitation) with those of deep-red/NIR multiscale optical imaging technologies (28-30) (enhanced sensitivity/contrast, multispectral capabilities), we initially monitored uptake, lymphatic drainage, and particle clearance in melanoma-bearing mice (31-34). These methods were extended to a larger-animal spontaneous melanoma miniswine model (29) to assess the feasibility of performing intraoperative image-guided metastatic disease detection, localization, and staging; to determine whether differences in nodal tumor burden could be sensitively discriminated; and to simulate more accurately the application of sentinel lymph node (SLN) biopsy procedures in humans.

\section{Results}

Nanoparticle design and characterization. From a clinical standpoint, modifying an inherently nontoxic and biocompatible material, amorphous silica, for targeted diagnostics and/or therapeutics offers an attractive strategy for ultimately achieving a favorable toxicologic profile in vivo, while, at the same time, preserving its versatility in a variety of patient settings. To realize such a platform, Cy5 dye-encapsulating core-shell silica nanoparticles (emission maxima, >650 nm), coated with methoxy-terminated polyethylene glycol (PEG) chains (PEG $\sim 0.5 \mathrm{kDa}$ ), were prepared according to previously published protocols (see Methods) $(10,27)$. The neutral PEG coating prevented uptake by other cells (opsonization). The use of bifunctional PEGs enabled attachment of small numbers of $\alpha_{v} \beta_{3}$ integrin-targeting cRGDY peptide ligands ( $\sim-7$ ligands per particle) (34) to maintain a small hydrodynamic size, facilitating efficient renal clearance.

Peptide ligands were labeled with the positron-emitting radionuclide ${ }^{124} \mathrm{I}$ through the use of a tyrosine linker to provide a signal that can be quantitatively imaged in 3 dimensions by PET ( ${ }^{124} \mathrm{I}$-cRGDY-PEG-ylated dots [ ${ }^{124} \mathrm{I}-\mathrm{cRGDY}-\mathrm{PEG}$-dots]; Figure 1A). An important practical advantage of relatively long-lived ${ }^{124} \mathrm{I}$

Table 1

Photophysical characterization using FCSA

\begin{tabular}{lccc} 
& $\begin{array}{c}\text { Hydrodynamic } \\
\text { radius } \mathbf{( n m )}\end{array}$ & $\begin{array}{c}\text { Brightness/ } \\
\text { particle }(\mathbf{k H z})\end{array}$ & $\begin{array}{c}\text { Concentration } \\
\mathbf{( m o l} / \mathbf{l})\end{array}$ \\
Free Cy5 dye & $0.67 \pm 0.008$ & 3.48 & $5.37 \times 10^{-4}$ \\
PEG-dots & $3.53 \pm 0.04$ & 10.91 & $6.61 \times 10^{-6}$ \\
cRGDY-PEG-dots & $3.40 \pm 0.04$ & 10.13 & $8.80 \times 10^{-6}$ \\
cRADY-PEG-dots & $3.25 \pm 0.04$ & 10.39 & $4.01 \times 10^{-6}$ \\
\hline
\end{tabular}

ATable 1 data were used in deriving the curves in Figure 1B. 
A
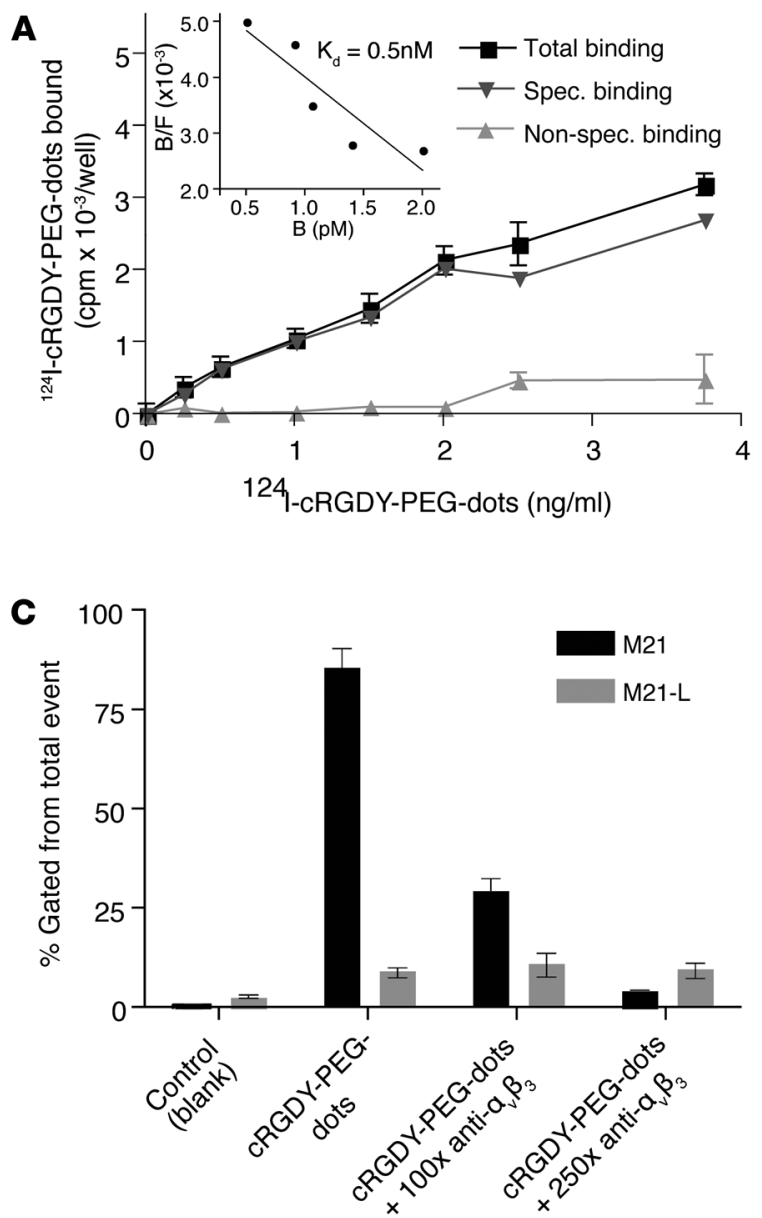

B

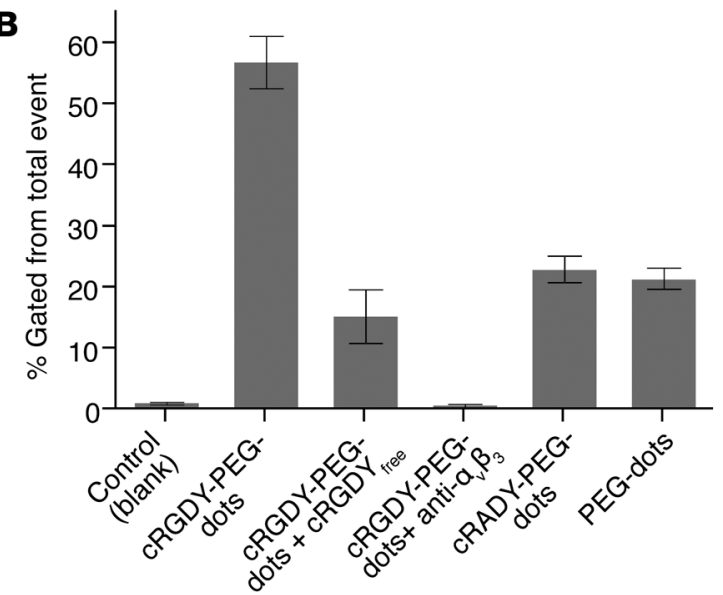

D

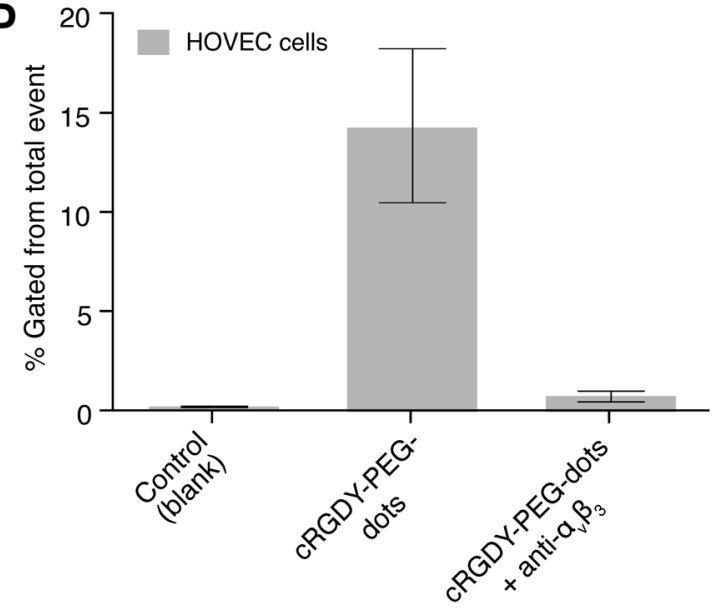

Figure 2

Competitive integrin receptor binding studies with ${ }^{124}$ I-cRGDY-PEG-dots, cRGDY peptide, and anti- $\alpha_{\nu} \beta_{3}$ antibody using 2 cell types. (A) High-affinity and specific binding of ${ }^{124} \mathrm{I}$-CRGDY-PEG-dots to M21 cells by $\gamma$-counting. The inset shows Scatchard analysis of binding data, plotting the ratio of the concentration of receptor-bound (B) to unbound (or free [F]) radioligand or the bound-to-free ratio (B/F) versus the receptor-bound receptor concentration; the slope corresponds to the dissociation constant, $K_{\mathrm{d}}$. (B) $\alpha_{v} \beta_{3}$ Integrin receptor blocking of M21 cells using flow cytometry and excess unradiolabeled cRGD or anti- $\alpha_{\nu} \beta_{3}$ antibody prior to incubation with cRGDY-PEG-dots and nonspecific binding with controls (RAD-PEG-dots, PEG-dots). (C) Specific binding of cRGDY-PEG-dots to M21 cells as against M21L cells lacking surface integrin expression using flow cytometry. Anti- $\alpha_{\nu} \beta_{3}$ integrin receptor antibody concentrations were used at 100 times (i.e., 100x) and 250 times (i.e., 250x) the particle (i.e., ${ }^{24}$ I-cRGDY-PEGdot) concentration. (D) Specific binding of cRGDY-PEG-dots to HUVECs by flow cytometry. Each bar represents mean \pm SD of 3 replicates.

(physical half-life, $4.2 \mathrm{~d}$ ) is that sufficient signal persists long enough to allow radiodetection up to at least several days after administration, when background activity has largely cleared, and tumor-to-background contrast is maximized. PEG- and cRADbound, PEG-coated particles containing tyrosine residues for ${ }^{124} \mathrm{I}$ labeling served as the control particle probes, the latter bearing a scrambled peptide (i.e., cyclo-[Arg-Ala-Asp-Tyr]). Purification of radiolabeled samples by size exclusion chromatography (Supplemental Figure 1; supplemental material available online with this article; doi:10.1172/JCI45600DS1) resulted in radiochemical yields of more than $95 \%$. Hydrodynamic diameters of approximately 7,6.8, and $6.5 \mathrm{~nm}$ (mean $\pm \mathrm{SD}, n=15$ ) were measured for cRGDY-PEG-dots, PEG-dots, and cRADY-PEG-dots, respectively, using fluorescence correlation spectroscopy (FCS) (Figure $1 \mathrm{~B}$ and Table 1). The relative brightness of the cRGDY-PEG-dots was determined to be approximately $200 \%$ greater than that of the free dye (Table 1), consistent with earlier results $(10,35)$.
Based on these physicochemical properties, we anticipated achieving a favorable balance between selective tumor uptake and retention versus clearance of the targeted particle, thus maximizing target-tissue localization while minimizing toxicity and normal-tissue radiation doses.

In vitro receptor-binding studies. To examine in vitro receptor-binding affinity and specificity of ${ }^{124} \mathrm{I}$-cRGDY-PEG-dots to melanoma and vascular endothelial cell surfaces relative to those of particle controls ( ${ }^{124} \mathrm{I}-\mathrm{PEG}-,{ }^{124} \mathrm{I}-\mathrm{cRADY}-\mathrm{PEG}$-dots), $\alpha_{v} \beta_{3}$ integrin-overexpressing (M21, HUVEC) and -nonexpressing (M21L) lines were used. Initially, M21 cells were incubated with increasing ${ }^{124} \mathrm{I}-\mathrm{cRGDY}-\mathrm{PEG}$-dot concentrations in the presence of excess nonradiolabeled cRGD peptide, and $\gamma$-counting was performed (Figure $2 \mathrm{~A}$ ). Scatchard analysis of the binding data yielded a dissociation equilibrium constant, $K_{\mathrm{d}}$, of $0.51 \mathrm{nM}$ (Figure $2 \mathrm{~A}$, inset) and a receptor concentration, $B_{\max }$, of $2.5 \mathrm{pM}$. Based on the $B_{\max }$ value, the $\alpha_{v} \beta_{3}$ integrin receptor density was estimated to be $1.0 \times 10^{4}$ 

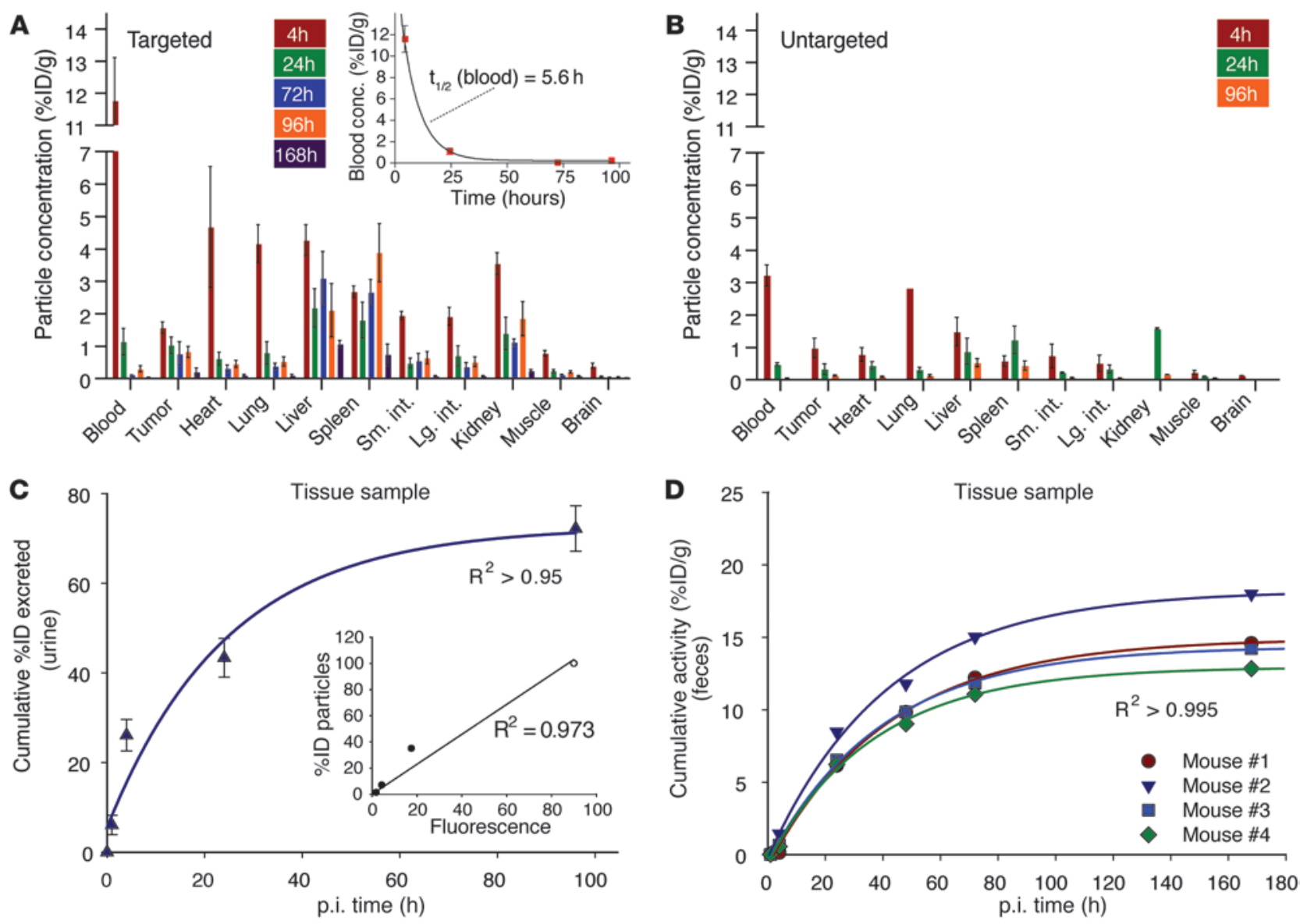

Figure 3

Pharmacokinetics and excretion profiles of the targeted and nontargeted particle probes. (A) Biodistribution of 124l-cRGDY-PEG-dots (targeted) in M21 tumor-bearing mice at various times from 4- to 168-hours p.i. The inset shows a representative plot of these data for blood to determine the $T_{1 / 2}$. Sm. int., small intestine; Lg. int., large intestine; conc., concentration. (B) Biodistribution of 124I-PEG-dots (untargeted) from 4- to 96-hours p.i. (C) Clearance profile of urine samples collected up to 168-hours p.i. of unradiolabeled cRGDY-PEG-dots $(n=3$ mice, mean \pm SD). The inset shows a strong correlation between the percentage of the injected particle dose excreted and the corresponding measured fluorescence signal. (D) Corresponding cumulative \%ID/g for feces at intervals up to 168-hours p.i. ( $n=4$ mice). For biodistribution studies, bars represent mean \pm SD.

integrin receptors per M21 cell, in reasonable agreement with the previously published estimate (36) of $5.6 \times 10^{4}$ integrin receptors per cell for this cell line. Incremental increases in integrin-specific M21 cellular uptake were also observed over a temperature range of $4^{\circ} \mathrm{C}$ to $37^{\circ} \mathrm{C}$, suggesting that cellular internalization contributed to overall uptake (data not shown).

Binding specificity of cRGDY-PEG-dots was further demonstrated with flow cytometry. Competitive binding assays showed complete blocking of receptor-mediated binding (Figure 2B) using anti- $\alpha_{v} \beta_{3}$ integrin antibody. No significant reduction was seen in the magnitude of receptor binding ( 10\% of M21) with M21L cells (Figure 2C) using either excess cRGDY peptide or anti- $\alpha_{v} \beta_{3}$ integrin antibody. These results were confirmed by additional $\gamma$-counting studies, and a 50\% binding-inhibition concentration, $\mathrm{IC}_{50}$, of $1.2 \mathrm{nM}$ was determined for the ${ }^{124} \mathrm{I}$-cRGDY-PEG-dot. This $\mathrm{IC}_{50}$ value suggests greater potency than that recently reported for engineered, high-affinity ${ }^{64} \mathrm{Cu}$-DOTA-knottin peptides ( $\mathrm{IC}_{50} \sim 20 \mathrm{nM}$ ) (37). Potency of cRGDY-PEG-dots was additionally evaluated by an anti-adhesion assay $(5,38)$ in order to assess particle multivalent interactions with $\alpha_{v} \beta_{3}$ integrin receptors. A multivalent enhance- ment factor of greater than 2.0 was determined for cRGDY-PEGdots relative to monomeric cRGD peptides after incubating each with M21 cells (data not shown). A final set of studies showed that, similar to M21 cells, excess antibody effectively blocked cRGDYPEG-dot receptor binding to HUVECs (Figure 2D).

Biodistribution and clearance studies. Biodistribution and blood, renal, and hepatobiliary clearance were evaluated by i.v. administering tracer doses ( $\sim 0.2$ nanomoles) of ${ }^{124} \mathrm{I}$-cRGDY-PEG-dots and ${ }^{124}$ I-PEG-dots to M21 tumor xenograft models. Although the percentage of the injected dose (ID) per gram tissue (\%ID/g) for the targeted probe was measured over a 196-hour postinjection (p.i.) time interval, comparison of the ${ }^{124} \mathrm{I}-\mathrm{cRGDY}-\mathrm{PEG}$-dot (Figure $3 \mathrm{~A}$ ) and ${ }^{124} \mathrm{I}-\mathrm{PEG}$-dot tracers (Figure $3 \mathrm{~B}$ ) was restricted to a 96-hour window, as in vivo activity for the latter was not detectable at 1 week p.i. Statistically significant $(P<0.05)$ differences in tracer uptake $(\% \mathrm{ID} / \mathrm{g})$ values were observed for blood, tumor, and major organs at 4 and 96 hours p.i. as well as at 24 hours p.i. for tumor and several other tissues (Supplemental Table 1). The targeted probe was almost entirely eliminated from the carcass at 1 week p.i. ( $3 \%$ of the ID remained). Tracer clearance half times 
Table 2

Radiation dosimetry for i.v.-injected ${ }^{124} \mid$-RGDY-PEG-dots and ${ }^{124 \mid}$-PEG-dots

\begin{tabular}{|c|c|c|c|c|c|c|c|c|}
\hline \multirow[b]{3}{*}{ Target organ } & \multicolumn{6}{|c|}{ Mouse } & \multicolumn{2}{|c|}{ Human ${ }^{A}$} \\
\hline & \multicolumn{3}{|c|}{ 124|-RGDY-PEG } & \multicolumn{3}{|c|}{ 124|-PEG } & \multirow{2}{*}{$\begin{array}{l}\text { 124|-RGDY-PEG } \\
\text { Absorbed dose } \\
\text { (cGy/mCi) }\end{array}$} & \multirow{2}{*}{$\begin{array}{l}\text { 124I-PEG } \\
\text { Absorbed dose } \\
\text { (cGy/mCi) }\end{array}$} \\
\hline & $T_{1 / 2}(\mathrm{~h})$ & $\begin{array}{c}\text { Activity, A } \\
(\% / D / g)^{B}\end{array}$ & $\begin{array}{l}\text { Absorbed dose } \\
\text { (cGy/mCi) }\end{array}$ & $T_{1 / 2}(\mathrm{~h})$ & $\begin{array}{c}\text { Activity, A } \\
(\% / D / g)^{B}\end{array}$ & $\begin{array}{l}\text { Absorbed dose } \\
\text { (cGy/mCi) }\end{array}$ & & \\
\hline Blood & 5.9 & 18.8 & 6.26 & 7.3 & 4.7 & 189 & - & - \\
\hline Heart wall & 6.8 & 7.0 & 266 & 34.1 & 0.8 & 120 & 0.31 & 0.087 \\
\hline Lungs & 8.5 & 5.7 & 267 & 37.7 & 3.0 & 498 & 0.30 & 0.26 \\
\hline Liver & 65.9 & 3.9 & 935 & 52.5 & 1.4 & 294 & 0.49 & 0.23 \\
\hline Spleen & 42.3 & 45.6 & 1,071 & 27.4 & 45.7 & 410 & 3.20 & 0.25 \\
\hline Small intestine wall & 30.3 & 1.8 & 251 & 13.2 & 0.9 & 61 & 0.30 & 0.12 \\
\hline \multirow[t]{2}{*}{ Large intestine wallC } & 23.9 & 2.0 & 228 & 49.4 & 0.5 & 99 & 0.43 & 0.21 \\
\hline & & & & & & & 0.72 & 0.42 \\
\hline Kidneys & 66 & 3 & 712 & 33 & 2.0 & 388 & 2.50 & 0.32 \\
\hline Muscle & 27.7 & 0.8 & 105 & 47.1 & 0.2 & 38 & 0.23 & 0.060 \\
\hline Brain & 13.9 & 0.4 & 29 & 8.5 & 0.2 & 8 & 0.19 & 0.15 \\
\hline TumorD & 73.5 & 1.5 & 380 & 37 & 0.9 & 146 & $\mathrm{~N} / \mathrm{A}$ & $\mathrm{N} / \mathrm{A}$ \\
\hline Adrenals & - & - & - & - & - & - & 0.40 & 0.083 \\
\hline Breasts & - & - & - & - & - & - & 0.14 & 0.042 \\
\hline Gall bladder wall & - & - & - & - & - & - & 0.29 & 0.097 \\
\hline Stomach wall & - & - & - & - & - & - & 0.27 & 0.065 \\
\hline Ovaries & - & - & - & - & - & - & 0.30 & 0.12 \\
\hline Pancreas & - & - & - & - & - & - & 0.39 & 0.081 \\
\hline Red marrow & - & - & - & - & - & - & 1.07 & 0.084 \\
\hline Osteogenic cells & - & - & - & - & - & - & 0.20 & 0.13 \\
\hline Skin & - & - & - & - & - & - & 0.16 & 0.038 \\
\hline Testes & - & - & - & - & - & - & 0.19 & 0.073 \\
\hline Thymus & - & - & - & - & - & - & 0.17 & 0.052 \\
\hline Thyroid & - & - & - & - & - & - & 0.19 & 0.043 \\
\hline Urinary bladder wall & - & - & - & - & - & - & 2.01 & 1.65 \\
\hline Uterus & - & - & - & - & - & - & 0.33 & 0.17 \\
\hline Total body & - & - & - & - & - & - & 0.034 & 0.075 \\
\hline $\begin{array}{l}\text { Effective dose equivalent } \\
\text { (cSv/mCi) }\end{array}$ & - & - & - & - & - & - & 0.86 & 0.26 \\
\hline $\begin{array}{l}\text { Effective dose } \\
\text { (cSv/mCi) }\end{array}$ & - & - & - & - & - & - & 0.60 & 0.23 \\
\hline
\end{tabular}

A70-kg standard human anatomic model. This is a "normal" (i.e., tumor-free) anatomic model and therefore does not yield tumor absorbed doses. Further, the OLINDA dosimetry program for human dosimetry includes cross-organ and "remainder-of-body" dose contributions and therefore yields absorbed-dose estimates even for organs for which time-activity data were not available. However, the OLINDA program does not include blood as a target region. In contrast, the mouse absorbed-dose calculation ignored cross-organ doses and therefore yielded absorbed-dose estimates only for target regions (organs and tumor) for which time-activity data were measured. B"\%ID/G" indicates the percentage of the ID per gram of tissue. CThe OLINDA dosimetry program for human dosimetry yields separate absorbed-dose estimates for the upper and lower large intestine walls, corresponding to the first and second entries, respectively, for "large intestine wall." DMouse melanoma model. "Activity, A," for both the targeted (124I-cRGDY-PEG-dots) and nontargeted (124I-PEG-dots) particles, refers to the zero-time intercept of the fitted time-activity concentration curve.

$\left(T_{1 / 2}\right)$ and bioavailability for blood, tumor, and major organs are shown in Table 2. For blood, the relatively long $T_{1 / 2}$ value (mean $\pm \mathrm{SD}$ ) for the ${ }^{124} \mathrm{I}$-PEG-dot tracer (i.e., $7.3 \pm 1.2$ hours), which is similar to that found for the nonradiolabeled particle control in normal mice by fluorescence detection (10), decreased to $5.6 \pm 0.15$ hours (Figure 3A, inset) for the ${ }^{124}$ I-cRGDY-PEG-dot tracer.

By appropriate organ mass-adjusted translation of the foregoing biodistribution data to humans, human normal organ radiation doses were derived and found to be comparable to those of other commonly used diagnostic radiotracers (Table 2, columns 8 and 9). Along with the findings of formal toxicity testing that the targeted probe was nontoxic at i.v. IDs 100 times the proposed human dose equivalent (based on standard body mass-normalized allometric scaling) and resulted in no tissue-specific pathologic effects (Supplemental Figure 2 and Supplemental Table 2), firstin-human targeted and nontargeted molecular imaging applications with these i.v.-injected agents are planned.

Efficient renal excretion was found for the approximately $7-\mathrm{nm}$ diameter targeted and nontargeted probes over a 168-hour time period by fluorimetric analyses of urine samples. Fluorescence signals were background corrected and converted to particle concentrations $(\% \mathrm{ID} / \mu \mathrm{l})$ based on a serial dilution calibration scheme (Figure 3C, inset; Supplemental Table 3, column 2; and ref. 10). Concentration values, along with age-dependent conservative estimates of the average urine excretion rate (39), permitted the cumulative excreted fluorescence signal (\%ID) to be computed (Supplemental Table 3, column 4). Nearly half of the ID was observed to be excreted over the first 24 hours p.i. and approxi- 

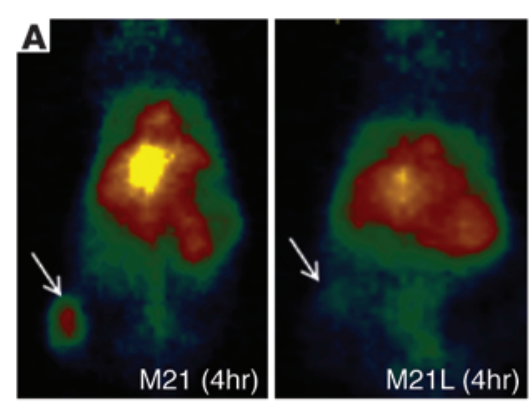

C

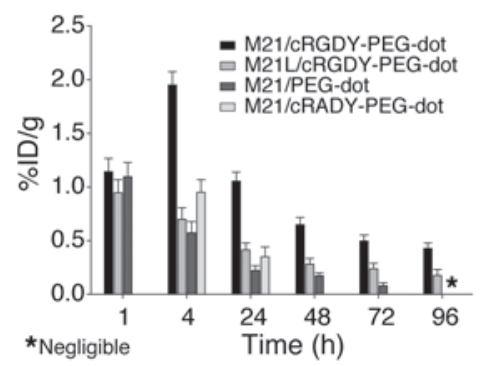

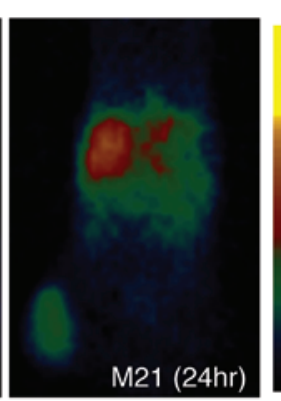

D

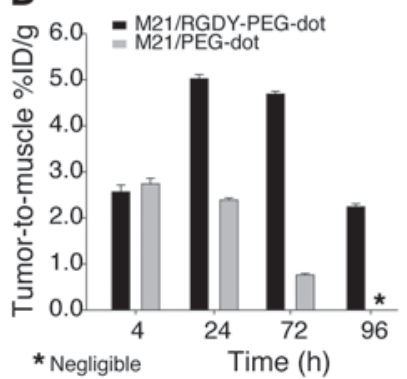

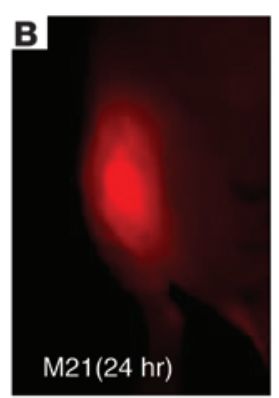

E

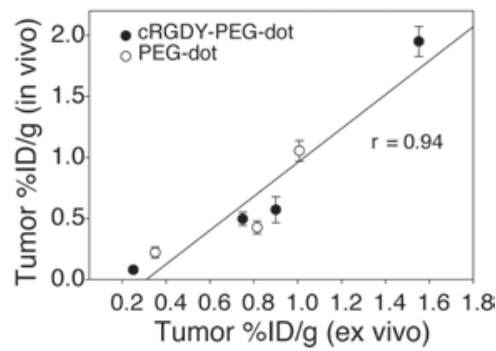

Figure 4

Serial in vivo PET imaging of tumor-selective targeting. (A) Representative whole-body coronal microPET images at 4-hours p.i., demonstrating M21 (left, arrow) and M21L (middle, arrow) tumor uptakes of 3.6 and $0.7 \% \mathrm{ID} / \mathrm{g}$, respectively, and enhanced M21 tumor contrast at 24 hours (right). (B) Representative 24-hour fluorescence image of the tumor. (C) In vivo uptake of ${ }^{124}$ l-cRGDY-PEG-dots in $\alpha_{v} \beta_{3}$ integrin-overexpressing M21 (black, $n=7$ mice) and nonexpressing M21L (medium dark gray, $n=5$ mice) tumors, ${ }^{124}$-PEG-dots in M21 tumors (dark gray, $n=5$ ), and 124I-cRAD-PEG-dots in M21 tumors (light gray, $n=3$ ). (D) M21 tumor-to-muscle ratios for ${ }^{124}$ I-cRGDY-PEG-dots (black) and ${ }^{124}$ I-PEG-dots (gray). (E) Correlation of in vivo and ex vivo M21 tumor uptakes of cRGDY-labeled and unlabeled probes. Each bar represents mean \pm SD.

mately $72 \%$ was excreted by 96 hours (Figure 3C), suggesting that the bulk of excretion occurred in the first day p.i. No significant particle fluorescence in urine could be detected 168 hours p.i. Fluorimetric determinations correlated strongly with corresponding $\gamma$-counter-derived \%ID values $\left(R^{2}=0.965\right.$, Supplemental Table 3 , column 5), suggesting that no significant free ${ }^{124} \mathrm{I}-\mathrm{cRGD}$ was present. In addition, as cumulative percentage dehalogenation values of urine samples by radio-TLC averaged less than $10 \%$ over a 24-hour period, RES trapping was not significantly underestimated (Supplemental Table 3, column 6). Fecal excretion profiles of the ${ }^{124} \mathrm{I}$-cRGDY-PEG-dot indicated that, on average, $7 \%$ and $15 \%$ of the ID was eliminated over 24 and 96 hours, respectively (Figure $3 \mathrm{D})$. To assess particle integrity, FCS analysis of urine specimens obtained prior to injection, and at 4 and 24 hours p.i. of the targeted probe, revealed that the particle was excreted intact and without release of the encapsulated dye (Supplemental Table 4); minor differences in solvent or surface chemistry likely contributed to the observed findings.

Serial whole-body PET studies. PET imaging of integrin expression in M21 and M21L subcutaneous hind leg xenograft mouse models was performed at multiple time points p.i. after i.v. injection of either ${ }^{124}$ I-cRGDY-PEG-dots or control particles (i.e., ${ }^{124} \mathrm{I}$-PEG-dots, ${ }^{124} \mathrm{I}$-cRAD-PEG-dots). Representative wholebody coronal microPET images at 4-hours p.i. of M21 and M21L tumors and at 24-hours p.i. of M21 tumor are shown in Figure 4A; a corresponding representative 24-hour fluorescence image of the tumor is shown in Figure 4B. The specific targeting of the $\alpha_{v} \beta_{3}$ integrin-overexpressing M21 tumor is clearly visible from these images. Average tumor \%ID/g and SDs are shown for the following groups (Figure 4C): M21 $(n=7)$ and M21L (control) tumors $(n=5)$ receiving the targeted ${ }^{124}$ I-cRGDY-PEG-dots; M21 tumor mice $(n=5)$ receiving nontargeted ${ }^{124}$ I-PEG-dots; and M21 mice $(n=3)$ receiving the ${ }^{124} \mathrm{I}-\mathrm{cRAD}-\mathrm{PEG}$-dots (Supplemental Figure $3 \mathrm{~A})$. At the time of maximum tumor uptake $(\sim 4$ hours p.i.), up to 3-fold higher activity concentrations (\%ID/g) were seen in M21 tumors than in controls. Differences were statistically significant at all time points p.i. $(P<0.05)$, except at 1 hour $(P=0.27)$. In vivo integrin receptor blocking studies of M21 tumors $(n=3)$ additionally confirmed particle binding specificity, with a $\sim 6$-fold decrease in tumor uptake in targeted tracer uptake before versus after administration of excess CRGD peptide (Supplemental Figure 3B).

Image-derived tumor-to-muscle uptake $(\% \mathrm{ID} / \mathrm{g})$ ratios for the ${ }^{124} \mathrm{I}$-cRGDY-PEG-dots revealed enhanced tumor contrast at later times ( 24-72 hours p.i.), while those for ${ }^{124}$ I-PEG-dots declined progressively with time (Figure 4D). This finding suggested that ${ }^{124}$ I-cRGDY-PEG-dots were tumor selective, which became more apparent as blood activity was cleared during the initial 24-hour period (compare Figure 4D with Figure 3A, inset). A statistically significant correlation was found between PET-derived tumor $\% \mathrm{ID} / \mathrm{g}$ values for both the targeted and nontargeted probes, and the corresponding ex-vivo $\gamma$-counter-derived tumor \%ID/g values (correlation coefficient $r=0.94, P<0.0016$; Figure 4E), confirming the accuracy of PET for noninvasively deriving quantitative biodistribution data.

Serial in vivo Cy5 fluorescence imaging and microscopy. In vivo fluorescence imaging studies were conducted using our targeted and nontargeted particle probes for mapping local/regional nodes and 


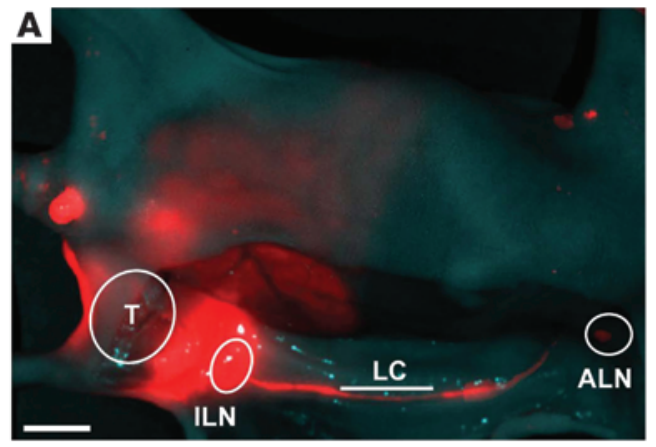

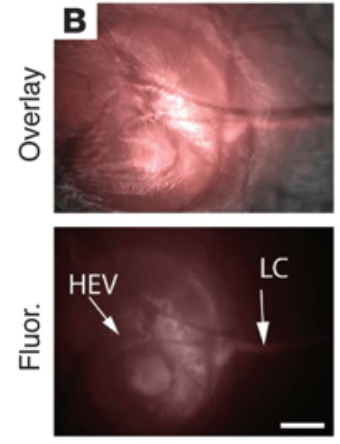

ILN

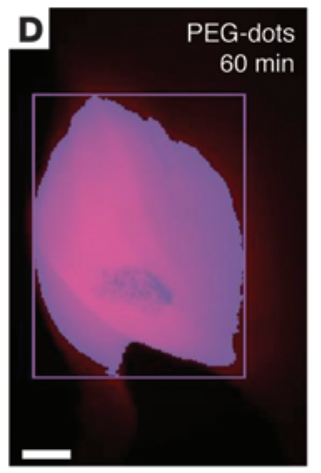

E
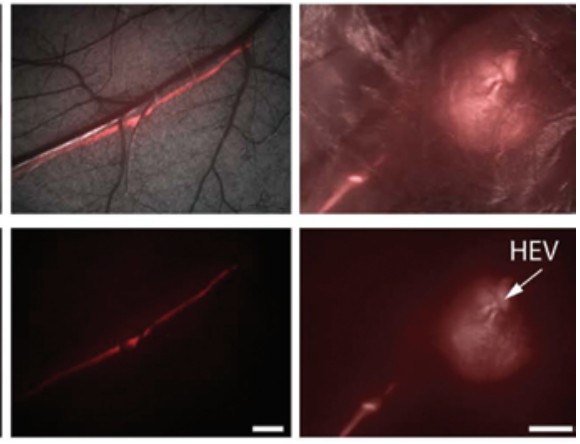

LC

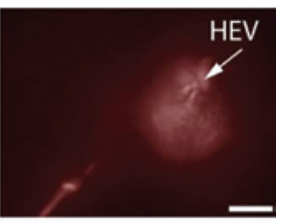

ALN
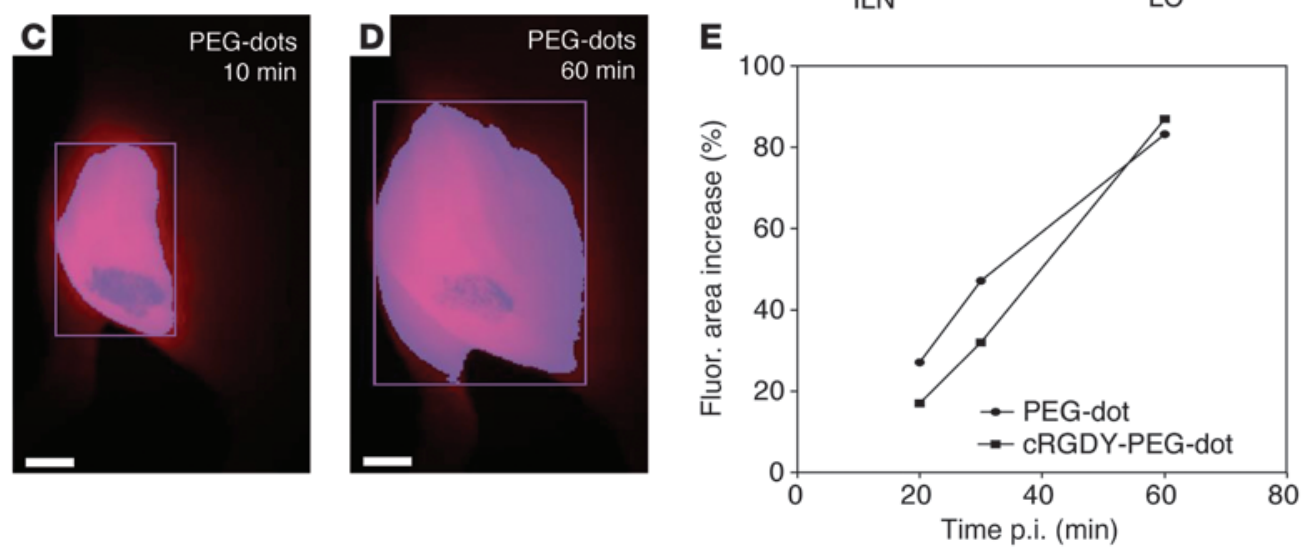

\section{Figure 5}

Nodal mapping using multiscale near-infrared optical fluorescence imaging. (A) Whole-body fluorescence imaging of the tumor site (T) and draining inguinal (ILN) and axillary (ALN) nodes and communicating lymphatics channels (LCs; bar) 1-hour p.i. in a surgically exposed living animal. (B) Corresponding coregistered white-light and high-resolution fluorescence images (top row) and fluorescence images only (bottom row), revealing nodal infrastructure of local and distant nodes, including high endothelial venules (HEVs). (C) Whole-body fluorescence image of the tumor site 10 minutes after subdermal PEG-dot injection. (D) Delayed whole-body fluorescence image of the tumor site 1 hour after PEG-dot injection. (E) Percentage increase in the area of fluorescence (fluor) relative to that measured at 10-minutes p.i. for targeted and nontargeted probes. Scale bars: $1.0 \mathrm{~cm}(\mathbf{A}) ; 500$ microns (B); $3 \mathrm{~mm}$ (C and D).

lymphatic channels in small-animal models, as the size of these structures (1-2 mm) may not be adequately resolved by PET imaging. Representative lymph node mapping was initially performed across spatial scales using the targeted probe, in conjunction with live-animal whole-body optical imaging (Figure 5A) and fluorescence microscopy techniques (Figure 5B), to visualize lymphatic drainage from the peritumoral region to the inguinal and axillary nodes in surgically exposed living animals. Peritumoral administration of the targeted probe revealed drainage into and persistent visualization of adjacent inguinal and popliteal nodes as well as more remote axillary nodes over a 4-hour interval with less than 1-mm resolution. Higher-resolution (i.e., submillimeter) fluorescence images (Figure 5B, bottom row) permitted more detailed intranodal architecture to be localized, including high endothelial venules, known to facilitate passage of circulating naive lymphocytes into the node; this may have important implications for nodal staging and the ability to detect micrometastases at earlier stages of disease. Smaller, less intensely fluorescent lymphatic branches were also visualized by fluorescence microscopy in the axillary region (data not shown). Thus, the smaller size of the targeted probe permitted the first draining, or sentinel, node proximal to the tumor to be visualized and also enabled detection of more distant nodes and visualization of the pattern of lymphatic drainage.
Differential rates of particle clearance were assessed for both nontargeted (PEG-dots) and targeted probes in intact, living mice bearing hind limb M21 xenografts versus those in controls. After subdermal, 4-quadrant, peritumoral injection, relatively rapid dispersion of both particle probes in M21 mice was observed over a 1-hour time period (measured against the initial 10-minute time point), with representative PEG-dot images shown (Figure 5, $\mathrm{C}$ and D). Using the fluorescence area increase (\%) as a measure of particle dispersion, no significant difference was seen between the targeted and nontargeted probes over the first hour p.i. (Figure $5 \mathrm{E})$. Further, in control animals, no percentage increase was found for either probe (data not shown). Although no fluorescent nodes were detected in intact mice, surgical exposure revealed lymphatic drainage from the inguinal region to the axilla for both probes, similar to that seen in Figure 5A.

Multimodal approaches were extended to a larger-animal spontaneous melanoma miniswine model (32) in order to assess the feasibility of performing real-time, intraoperative image-guided metastatic disease detection and staging and to determine whether tumor burden could be sensitively discriminated with correlative histology. After i.v. injection, whole-body dynamic ${ }^{18} \mathrm{~F}$-fluorodeoxyglucose ( $\left.{ }^{18} \mathrm{~F}-\mathrm{FDG}\right)$ PET-CT scanning identified an ${ }^{18} \mathrm{~F}-\mathrm{FDG}-$ avid melanomatous lesion adjacent to the spine on the upper back 


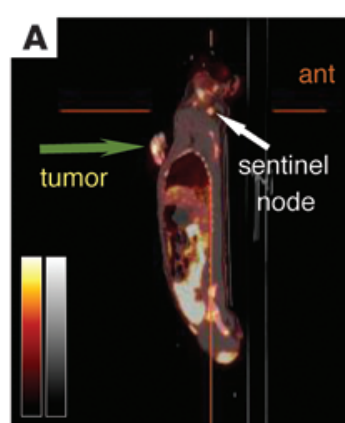

Sagittal

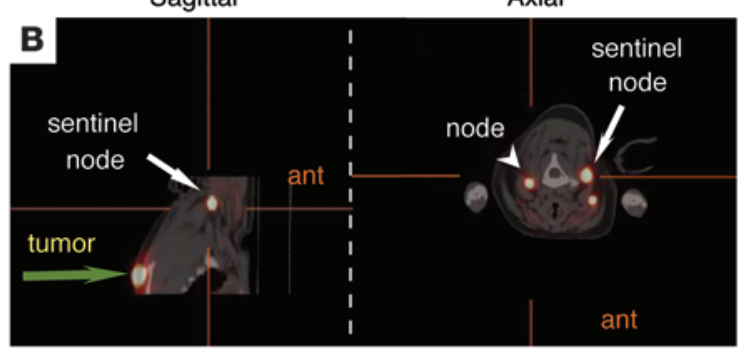

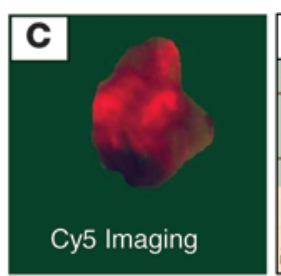
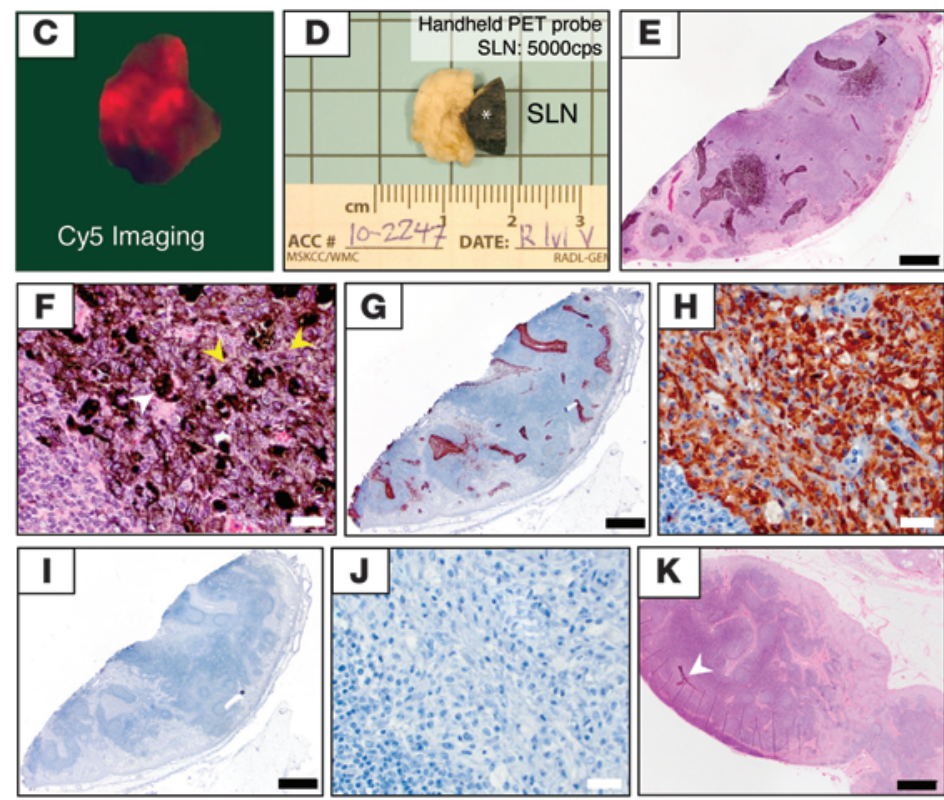

\section{Figure 6}

Imaging of metastatic disease in a spontaneous melanoma miniswine model. (A) Whole-body dynamic ${ }^{18} \mathrm{~F}$-FDG PET-CT sagittal and axial views, demonstrating primary tumor (green arrow) and single SLN (white arrow) posteriorly within the right (Rt) neck after i.v. injection. ant, anterior. (B) High-resolution dynamic PET-CT scan 1 hour after subdermal, 4-quadrant, peritumoral injection of ${ }^{124}$ |-RGD-PEG-dots (SLN, arrow; left-sided node, arrowhead). (C) Whole-body Cy5 fluorescence image of the excised SLN. (D) Gross image of the cut surface of the black-pigmented SLN (asterisk), which measured $1.3 \times 1.0 \times 1.5 \mathrm{~cm}^{3}$, and annotated $\gamma$ counted activity. (E) Low-power view of H\&E-stained SLN, demonstrating scattered melanomatous clusters. (F) Corresponding high-power view of H\&E-stained SLN, revealing melanoma cells (yellow arrowheads) and melanophages (white arrowhead). (G) Low-power image of a melanoma-specific marker, HMB-45, in representative SLN tissue. (H) High-power image of HMB-45-stained SLN tissue. (I) Low-power image of representative normal porcine nodal tissue. (J) High-power image of representative normal porcine nodal tissue. (K) Low-power view of H\&E-stained contralateral hypermetabolic lymph node, demonstrating scattered melanomatous clusters (arrowhead). Tumor burden in this smaller node $\left(1.0 \times 0.6 \times 1.0 \mathrm{~cm}^{3}\right)$ was estimated to be 10 - to 20 -fold less than that in the SLN by pathological analysis. Scale bars: $1 \mathrm{~mm}(\mathbf{E}, \mathbf{G}, \mathbf{I}$, and $\mathbf{K}) ; 20 \mu \mathrm{m}(\mathbf{F}, \mathbf{H}$, and J).

as well as a hypermetabolic right-sided SLN in the posterior neck (Figure 6A, white arrow). Subdermal, 4-quadrant peritumoral injection of ${ }^{124} \mathrm{I}$-RGD-PEG-dots 48 hours later confirmed the ${ }^{18} \mathrm{~F}$ FDG imaging findings and identified 2 additional hypermetabolic nodes, 1 within the left posterior neck (Figure 6B, arrowhead) and the second immediately anterior to the SLN 5-minutes and 1-hour p.i. by dynamic PET scanning. No additional PET-avid nodes or other suspicious areas of increased uptake were seen on a 1-hour whole-body PET scan. Corresponding optical imaging of the excised right-sided SLN demonstrated fluorescence signal within the black-pigmented (melanin-containing) gross specimen (Figure $6, C$ and D), which measured $1.3 \times 1.0 \times 1.5 \mathrm{~cm}^{3}$, as compared with the smaller posterior left-sided hypermetabolic node, which measured $1.0 \times 0.6 \times 1.0 \mathrm{~cm}^{3}$ (Supplemental Figure 4A). Significantly increased background-corrected SLN activity, detected with a hand-held intraoperative positron probe, corroborated these optical findings (Figure 6D, annotation).

H\&E-stained tissue sections from the SLN showed scattered, dark melanomatous clusters on low-power views (Figure 6E), which were found to comprise both melanoma cells and melanincontaining macrophages (i.e., melanophages) on high-power views (Figure 6F), similar to findings for excised primary lesions (data not shown). Immunohistochemical staining of the SLN with a known human melanoma marker, HMB45, demonstrated positive expression of this marker on low-power views (Figure 6G) and high-power views (Figure 6H). Representative normal-appearing porcine nodal tissue harvested from the neck revealed no metastatic infiltration in representative low-power views (Figure 6I) and high-power views (Figure 6J). By contrast, low-power views (Supplemental Figure 4B and Figure 6K) of H\&E-stained sections from the posterior left-sided hypermetabolic node demonstrated a few smaller-sized melanomatous clusters containing melanoma cells and melanophages (Supplemental Figure 4C). Tumor burden in this smaller node, estimated to be 10- to 20-fold less than in the SLN by pathological analysis, was sensitively discriminated by the targeted particle probe.

\section{Discussion}

Here, we report on an ultrasmall, high-affinity, and efficiently cleared silica nanoparticle probe, which has been recently approved for first-in-human clinical trials and which successfully overcomes a number of the limitations of other particle platforms. This multimodal platform has advanced to the point of clinical translatability. Its applications include real-time, intraoperative detection and imaging of nodal metastases, differential tumor burden, and lymphatic drainage patterns in melanoma. Although several investigators have synthesized radiolabeled fluorescent particle probes $(25,30,40)$, our multimodal agent has been radiolabeled with the long-lived positron-emitter iodine-124, and, thus, we believe it can provide unique longer-term pharmacokinetic clearance and 
targeting information over the course of days. The complementary nature of this platform, coupled with its small size $(\sim 7-\mathrm{nm}$ i.d.), may facilitate clinical assessments by enabling the seamless integration of imaging data acquired at different spatial, temporal, and sensitivity scales, potentially providing new insights into fundamental molecular processes governing tumor biology.

The results of this study underscore the clear-cut advantages offered by PET. Using this quantitative and highly sensitive imaging tool, we were able to noninvasively extract an accurate, reproducible, and comprehensive body of data for the targeted probe: (a) molecular information, including receptor expression levels, binding affinity, and specificity; (b) in vivo distribution and targeting kinetics; (c) clearance and dehalogenation profiles; (d) blood/tissue residence times and bioavailability; and (e) radiation dosimetry.

Our in vitro results show receptor-binding specificity of the 7-nm-targeted particle probe to M21 cells and HUVECs. Similar findings have been reported with receptor-binding assays using the same cell types but with the monovalent form of the peptide (36). Importantly, the multivalency enhancement of the cRGDYbound particle probe, along with the extended blood and tumor $T_{1 / 2}$ values, are key properties associated with the particle platform that are not found with the monovalent form of the peptide.

The integrin-binding peptide, cRGD, a well-established integrinbinding molecular marker, was selected to elucidate the biological and kinetic properties of the peptide-bound particle and additionally enable the biological performance of our multimodal platform to be benchmarked against other cRGD agents (i.e., peptide tracers). However, another more tumor-specific, high-affinity ligand could be investigated as the targeting moiety in future studies. The biological properties of particle-based systems are generally quite different from those of simple (i.e., small monovalent) targeting peptides, and the choice of the proper targeting agent (i.e., molecular versus particle-based probes) in the clinical setting will rest, in part, on the application of interest, the tumor type/composition, and standard-of-care considerations.

The relatively long blood $T_{1 / 2}$ for the ${ }^{124} \mathrm{I}-\mathrm{PEG}$-dot tracer may be a consequence of the chemically neutral PEG-coated surface, rendering the probe biologically inert and significantly less susceptible to phagocytosis by the RES. However, recognition of the ${ }^{124}$ I-cRGDYPEG-dot tracer by target integrins and/or more active macrophage activity may have led to reductions in the $T_{1 / 2}$ value. These values, however, are substantially longer than published blood $T_{1 / 2}$ values of existing cRGDY peptide tracers ( 13 minutes) (5), potentially leading to increased probe bioavailability, facilitating tumor targeting, and yielding higher tumor uptake over longer time periods. Moreover, the tumor $T_{1 / 2}$ for the ${ }^{124} \mathrm{I}$-cRGDY-PEG-dot was found to be 13-times greater than that for blood, compared with only a 5-fold difference for the ${ }^{124}$ I-PEG-dot, suggesting substantially greater target-tissue localization of the former than the latter. Such mechanistic interpretations of the in vivo data can be exploited clinically to refine diagnostic, treatment-planning, and treatment-monitoring protocols.

The greater accumulation in and slower clearance from M21 tumors, relative to that of surrounding normal structures, allows discrimination of specific tumor uptake mechanisms from nonspecific mechanisms (i.e., tissue perfusion, leakage) in normal tissues. However, a small component of the M21 tumor uptake can presumably be attributed to vascular permeability alterations (i.e., enhanced permeability and retention effects) (41), largely reflected in the observed \%ID/g increases for the control tracer ( ${ }^{124} \mathrm{I}-\mathrm{PEG}$ - dots, Figure 4C) at earlier p.i. time points. At 1-hour p.i., no significant \%ID/g increases were seen in the M21 tumors over the controls. This observation may represent the effects of differential perfusion in the first hour, with tumor accumulation and retention primarily seen at later p.i. times (i.e., 24 hours). Further, in comparison with those of the clinically approved peptide tracer, ${ }^{18} \mathrm{~F}$-galacto RGD $(42,43)$, nearly 2 -fold greater maximum uptake values were found in M21 tumors for the targeted dots at 2-hours p.i. (data not shown), while additionally offering advantages of multivalent binding and extended circulation times.

The advantages of the combined optical-PET probe highlight the versatility of the platform for in vivo applications. This is particularly true in small-animal models, in which the ability to assess anatomic structures having sizes at or well below the resolution limit of the PET scanner (i.e., the so-called partial-volume effect) may undermine detection and quantitation of activity in lesions. In these cases, assessment of metastatic disease in small local/regional nodes, important clinically for melanoma staging and treatment, may not be adequately resolved by PET imaging, given that the size of the nodes we typically observed was on the order of the PET spatial resolution (1-2 mm). By exploiting the significantly improved photophysical features of encapsulated dyes, such as Cy5, as well as the enhanced detection sensitivity and contrast achievable at longer emission wavelengths, detailed information pertaining to the localization of superficial nodes, lymphatic function, and clearance can be acquired using deepred/NIR fluorescence imaging strategies.

Larger-animal spontaneous melanoma models may more accurately reflect human disease and enable improved simulation of surgical procedures used in humans (i.e., SLN mapping). Locally injected ${ }^{124}$ I-cRGDY-PEG-dot tracer and dynamic PET imaging enabled superior detection sensitivity and discrimination of metastatic tumor burden within hypermetabolic neck nodes compared with the PET imaging agent, ${ }^{18} \mathrm{~F}-\mathrm{FDG} .{ }^{18} \mathrm{~F}-\mathrm{FDG}$ is an indicator of glucose metabolism, accumulating within metabolically active tumors that use this substrate. Traditionally used to stage clinical melanoma, it failed to accurately stage nodal disease in this representative miniswine. A number of well-known limitations are associated with whole-body ${ }^{18}$ F-FDG PET (44), particularly when imaging patients with early-stage melanoma, including a low mean sensitivity of $17.3 \%(0 \%-40 \%)$ for detecting SLNs and an inability to detect micrometastases less than about $1-\mathrm{cm}$ i.d. In the present study, the ${ }^{124} \mathrm{I}-\mathrm{cRGDY}-\mathrm{PEG}$-dot tracer was able to discriminate at least order of magnitude differences in metastatic tumor burden (i.e., 10- to 20-times greater) between the bilateral neck nodes, as determined by high-power microscopy, while ${ }^{18}$ F-FDG PET could not. Although metastatic disease was detected within the SLN, tumor was missed within the smaller $(1.0 \times 0.6 \times$ $1.0 \mathrm{~cm}^{3}$ ) contralateral node.

Thus, for metastatic disease assessment, the use of dynamic PET imaging as a depth-sensitive, volumetric imaging tool, in conjunction with ${ }^{124}$ I-cRGDY-PEG-dots, will offer distinct advantages in large-animal models in the intraoperative setting. Superior detection sensitivity, metabolic nodal status, and enhanced penetration for the mapping of deep-seated nodal and tissue activities may complement extended real-time fluorescence imaging assessments of metastatic tumor burden in the future. The use of PET will be critical for confirming the findings of depth-insensitive optical imaging tools, particularly in anatomic regions associated with unpredictable patterns of metastatic disease spread. 


\section{Methods}

Synthesis of cRGDY-PEG-dots, cRADY-PEG-dots, and PEG-dots. Particles were prepared by a modified Stöber-type silica condensation $(35,45-47)$. Reactive derivatives of the organic dye Cy5, exhibiting emission maxima about $650 \mathrm{~nm}$, were used to produce particles containing 2 dye equivalents within the particle core. Tyrosine residues were conjugated to PEG chains $(48,49)$ for attachment of radioiodine or stable iodine moieties. All samples were optical density matched at their peak absorption wavelength $(640 \mathrm{~nm})$ prior to radiolabeling. cRGDY peptides containing the sequence cyclo-(Arg-Gly-Asp-Tyr) and cRADY peptides containing the sequence cyclo-(Arg-Ala-Asp-Tyr) and bearing cysteine residues (Peptide International) were attached to functionalized PEG chains via a cysteine-maleimide linkage. The number of cRGD ligands per nanoparticle was empirically calculated from known FCS-derived nanoparticle concentrations and starting concentrations of cRGD-PEG-silane derivatives, assuming that the surface functionalization reaction completely consumed all bifunctional PEGylation reagent as an upper bound.

Mechanism of PEG attachment to the $C$ dot surface. Bifunctional PEGs, MALdPEG12-NHS ester (Quanta Biodesigns Ltd.), were derivatized with silanes, specifically 3-aminopropyl triethoxysilane (Gelest), for attachment to the silica surface and for peptide coupling via reactions between the sulfhydryl groups and maleimide moieties of the derivatized PEGs. In addition, methoxy-capped PEG chains were added to the particle surface using functional organosilicon compounds ( $\mathrm{Si}$ compounds), specifically ( $\mathrm{MeO})_{3} \mathrm{Si}-\mathrm{PEG}$ (Gelest), according to modified protocols (40). Briefly, $(\mathrm{MeO})_{3} \mathrm{Si}-\mathrm{PEG}$ was added, at approximately 3 molar excess, to particles in a water/alcohol basic mixture $(\sim 1: 5 \mathrm{v} / \mathrm{v})$, and the mixture was stirred overnight at room temperature.

Hydrodynamic size and relative brightness comparison measurements by FCS. The hydrodynamic radius, brightness, and concentrations of cRGDY-PEG-, cRADY-PEG-, and PEG-dots, as against free Cy5 dye, were initially determined by dialyzing these particle samples to water, diluting into physiological saline $\left(0.15 \mathrm{M} \mathrm{NaCl}\right.$ in $\left.\mathrm{H}_{2} \mathrm{O}\right)$, and analyzing the resulting specimens on a Zeiss LSM 510 Confocor 2 FCS using HeNe 633-nm excitation (46). The instrument was calibrated with respect to particle size prior to all measurements. Average hydrodynamic sizes of the dye and particle species were estimated based on diffusion time differences, while relative differences in brightness were assessed using count rates per molecule per particle.

To assess particle integrity in urine specimens, size and brightness measurements were determined prior to and at 4- and 24-hour after i.v. injection of $200 \mu$ liodinated ${ }^{127}$ I-cRGDY-PEG-dots $(n=3$ specimens per time point). Three separate runs were performed per specimen to derive average particle sizes and SDs. Controls for urine analyses were prepared by mixing known volumes of particles with specimens collected from control mice.

Radiolabeling of $C$ dot conjugates. Radiolabeling of the PEG- and cRGDYPEG-dots was performed using the IODOGEN method (Pierce) $(10,50)$. Specific activities of the ${ }^{124} \mathrm{I}$-bound particle fractions eluted from PD-10 columns were $\sim 300-1,000 \mathrm{mCi} / \mu \mathrm{m}$, assessed using a dose calibrator (Capintec) and radio-TLC. TLC plates were developed using a mixture of acetic $\mathrm{acid} / \mathrm{methanol}(80: 20 \mathrm{v} / \mathrm{v})$ and dried, and the absorbance was analyzed by a TLC plate reader (Bioscan). Particle concentrations were based on fluorescence spectrometer measurements (Varian Inc.) at maximum excitation/ emission wavelengths of 650 and $680 \mathrm{~nm}$, respectively.

Cells and cell culture. Human melanoma M21 and M21 variant (M21-L, $\alpha_{v}$ negative) cell lines were obtained from D.A. Cheresh (University of California San Diego, San Diego, California, USA). Cells were maintained in RPMI 1640 media/10\% fetal BSA, and $2 \mathrm{mM} \mathrm{L-glutamine,} \mathrm{penicillin,}$ and streptomycin (Core Media Preparation Facility, Memorial SloanKettering Cancer Center). HUVECs, cultured in M199 media/10\% fetal bovine serum, $20 \mu \mathrm{g} / \mathrm{ml}$ endothelial cell growth factor, and $50 \mu \mathrm{g} / \mathrm{ml}$ heparin, penicillin, and streptomycin, were provided by S. Rafii, Weill Cornell Medical Center, New York, New York, USA.
In vitro cell-binding and molecular specificity of ${ }^{124} I-c R G D-P E G$-dots. To assay particle binding and specificity for M21 cells, 24-well plates were coated with $10 \mu \mathrm{g} / \mathrm{ml}$ collagen type I (BD Biosciences) in PBS and incubated $\left(37^{\circ} \mathrm{C}, 30\right.$ minutes $)$. M21 cells $\left(3.0 \times 10^{5}\right.$ cells/well to $4.0 \times 10^{5}$ cells/well $)$ were grown to confluency and washed with RPMI 1640 media/0.5\% BSA. ${ }^{124} \mathrm{I}$-cRGD-PEG-dots $(0-4.0 \mathrm{ng} / \mathrm{ml})$ were added to wells, and cells were incubated $\left(25^{\circ} \mathrm{C}\right.$, 4 hours), washed with RPMI 1640 media/0.5\% BSA, and dissolved in $0.2 \mathrm{M} \mathrm{NaOH}$. Radioactivity was assayed using a 1480 Automatic Gamma Counter (Perkin Elmer) calibrated for iodine-124. Nonspecific binding was determined in the presence of a 1,000-fold excess of cRGD (Peptides International). Scatchard plots of the binding data were generated and analyzed using linear regression analyses (Microsoft Excel 2007) to derive receptor-binding parameters $\left(K_{\mathrm{d}}, B_{\max }, \mathrm{IC}_{50}\right)$.

In vitro cell-binding studies using optical detection methods. Differential binding of cRGDY-PEG-dots and PEG-dots to M21 cells was evaluated over a range of incubation times (up to 5 hours) and particle concentrations $(0-8 \mathrm{ng} / \mathrm{ml})$ using flow cytometry. After incubation, cells $\left(3.0 \times 10^{5}\right.$ cells/well $)$ were washed with RPMI 1640 media/0.5\% BSA, detached using $0.25 \%$ trypsin/EDTA, pelleted in a microcentrifuge tube ( 5 minutes at $153 \mathrm{~g}, 25^{\circ} \mathrm{C}$ ), resuspended in BD FACSFlow solution (BD Biosciences), and analyzed in the $\mathrm{Cy} 5$ channel to determine the percentage of particle-bound probe (FACSCalibur, Becton Dickinson). The particle concentration (i.e., $\sim 2.0 \mathrm{ng} / \mathrm{ml}$ ) and incubation time (i.e., 4 hours) yielding maximum differential binding were used for subsequent competitive binding assays and specificity studies.

Competitive binding studies were performed after incubation of cRGDYPEG-dots with M21 cells, M21L cells, and HUVECs in the presence of excess cRGD and/or mouse monoclonal anti-human integrin $\alpha_{v} \beta_{3}$ fluorescein-conjugated antibody (Millipore) and analyzed by flow cytometry. In addition, particle control binding studies (cRADY-PEG-dots and PEGdots) were conducted with $\mathrm{M} 21$ cells at concentrations similar to those used for cRGDY-PEG-dots ( $1 \mathrm{mM})$. To assess potency of the RGDY-PEG dots relative to that of the cRGD peptide, an anti-adhesion assay was used $(5,38)$. Ninety-six-well microtiter plates were coated with vitronectin in PBS $(5 \mu \mathrm{g} / \mathrm{ml})$, followed by $200 \mu \mathrm{l} \mathrm{RPMI} / 0.5 \%$ BSA $\left(1\right.$ hour, $\left.37^{\circ} \mathrm{C}\right)$. Cells $\left(3 \times 10^{4}\right.$ cells $/ 100 \mu \mathrm{l} /$ well $)$ were incubated in quadruplicate $(30$ minutes, $25^{\circ} \mathrm{C}$ ), with various concentrations of cRGDY-PEG-dots or cRGD peptide in RPMI/0.1\% BSA, and added to vitronectin-coated plates (30 minutes, $37^{\circ} \mathrm{C}$ ). Wells were rinsed with RPMI/0.1\% BSA to remove nonadherent cells. Adherent cells were fixed with $4 \% \mathrm{PFA}\left(20\right.$ minutes, $\left.25^{\circ} \mathrm{C}\right)$ and stained with methylene blue $\left(1\right.$ hour, $\left.37^{\circ} \mathrm{C}\right)$ for determination of optical densities using a Tecan Safire plate reader $\left(\lambda_{\mathrm{ex}}=650 \mathrm{~nm}, \lambda_{\mathrm{em}}=680 \mathrm{~nm}\right)$. The multivalent enhancement factor was computed as the ratio of the CRGD peptide-to-cRGDY-PEG-dot $\mathrm{IC}_{50}$ values (5).

Animal models and tumor inoculation. All animal experiments were done in accordance with protocols approved by the Institutional Animal Care and Use Committee of Memorial Sloan-Kettering Cancer Center and followed NIH guidelines for animal welfare. Male athymic $n u / n u$ mice (6-8 weeks old, Taconic Farms Inc.) were provided with water containing potassium iodide solution to block thyroid gland uptake of free radioiodine and maintained on a Harlan Teklad Global Diet 2016, ad libitum (10). M21 or M21L xenografts were generated by coinjecting equal volumes of cells $\left(\sim 5 \times 10^{6}\right.$ cells/100 $\mu \mathrm{l}$ ) and Matrigel subcutaneously into the hind legs of different mice. Average tumor volumes of $200 \mathrm{~mm}^{3}$ were used for all studies.

In vivo pharmacokinetic and $T_{1 / 2}$ measurements. The $\% \mathrm{ID} / \mathrm{g}$ values, corrected for radioactive decay to the time of injection, were measured by sacrificing groups of mice at specified times after i.v. injection of ${ }^{124}$ I-cRGDY-PEGdots or ${ }^{124} \mathrm{I}$-PEG-dots $(\sim 20 \mu \mathrm{Ci} /$ mouse $)$ and harvesting, weighing, and counting blood, tumor, and organs in a scintillation $\gamma$-counter. The resulting TAC data for each tissue were fit to a decreasing monoexponential 
function to estimate values of the tissue/organ $T_{1 / 2}$ and the zero-time intercept of the fitted time-activity concentration curve (determined for both the targeted [124I-cRGDY-PEG-dots] and nontargeted [124I-PEG-dots] particles), respectively, of the function.

In vivo excretion studies. The fraction of cRGDY-PEG-dots excreted in the urine over time was estimated by fluorimetry (10) over a 168-hour period ( $n=3$ mice/time point) and $\gamma$-counting. Briefly, mice were i.v. injected with either $200 \mu$ l $^{127}$ I-cRGDY-PEG-dots or ${ }^{127}$ I-PEG-dots for fluorimetric studies. Particle concentrations at each time point were determined on the basis of serial dilution calibration curves (10). Concentration values, along with estimates of average daily mouse urine volumes, were used to compute cumulative \%ID urine excreted. \%ID values were correlated with the corresponding decay-corrected cumulative excreted radioactivity (\%ID) at 1-, 4-, and 24- hours p.i. of ${ }^{124}$ I-cRGDY-PEG-dots. To assess cumulative fecal excretion, feces were collected in metabolic cages over similar time intervals after injection of $200 \mu \mathrm{l}^{124} \mathrm{I}$-cRGDY-PEG-dots ( $n=4 \mathrm{mice} /$ time point), and specimen activities were determined using a $\gamma$-counter.

Cumulative percentage dehalogenation was assessed by radio-TLC analysis of urine samples using $10 \%$ acetic acid/methanol as a running buffer. Plates were dried and analyzed with a Bioscan System 200 Imaging Scanner; individual peaks were separated, and areas under the curve were calculated using system software.

Dosimetry. Time-activity functions derived for each tissue were analytically integrated (with inclusion of the effect of radioactive decay) to yield the corresponding cumulative activity (i.e., the total number of radioactive decays). ${ }^{124}$ I mouse organ absorbed doses were then calculated by multiplying the cumulative activity by the ${ }^{124} \mathrm{I}$ equilibrium dose constant for nonpenetrating radiations (positrons) (51), assuming complete local absorption of such radiations and ignoring the contribution of penetrating radiations (i.e., $\gamma$-rays). The mouse normal organ cumulated activities were converted to human normal organ cumulated activities by adjustment for the differences in total-body and organ masses between mice and humans (assuming 70-kg standard human) (52). The human normal organ cumulated activities calculated were entered into the OLINDA dosimetry computer program to calculate, using the formalism of the Medical Internal Dosimetry Committee of the Society of Nuclear Medicine $(53,54)$, the standard human organ absorbed doses.

Single-dose toxicity testing and histopathology of ${ }^{127} I-c R G D Y-P E G-$ dots. Toxicity testing was performed in 6 groups of male and female B6D2F1 mice ( 8 weeks old, The Jackson Laboratory) at doses 100 times the proposed human dose equivalent. The treatment group ( $n=6$ males, $n=6$ females) received i.v.-injected targeted probe (i.e., ${ }^{127} \mathrm{I}$-cRGDY-PEG-dots) at a dose of $1 \times 10^{-9} \mathrm{moles} / \mathrm{animal}$, while the control group ( $n=6$ males, $n=6$ females) received the same dose of ${ }^{127}$ I-PEG-dots (vehicle) in a single i.v. injection $(200 \mu \mathrm{l})$. Untreated controls ( $n=2$ males, $n=2$ females) were additionally tested. Mice were observed daily over 14 days p.i. for signs of morbidity/mortality and weight changes. Gross necropsy, histopathology, and blood sampling for hematology and serum chemistry evaluation were performed at 7 and 14 days p.i.

Serial PET imaging of tumor-specific targeting. Imaging was performed using a dedicated small-animal PET scanner (Focus 120 microPET; Concorde Microsystems). Mice bearing M21 or M21L hind leg tumors were anesthetized using $2 \%$ isoflurane anesthesia in oxygen during the scan period. One-hour list-mode acquisitions were initiated after i.v. injection of $200 \mu \mathrm{Ci}{ }^{124} \mathrm{I}-\mathrm{cRGDY}$ PEG-dots or ${ }^{124} \mathrm{I}-\mathrm{PEG}$-dots in all mice, followed by serial 30-minute static images over a 96-hour interval. ${ }^{124} \mathrm{I}-\mathrm{cRADY}-\mathrm{PEG}-\mathrm{dot}(200 \mu \mathrm{Ci})$ static images were additionally acquired at 4- and 24-hours after i.v. injection. Acquisition of whole-body optical fluorescence images at 2 -nm intervals from $630 \mathrm{~nm}$ to $850 \mathrm{~nm}$, using a 575- to 605-bandpass excitation filter and a 645-nm longpass emission filter (Maestro, Cambridge Research Instruments), was performed and spectrally deconvolved as previously described (10).
PET image reconstruction and analysis. Voxel count rates in the reconstructed images were decay corrected and converted to activity concentrations (percentage $\% \mathrm{ID} / \mathrm{g}$ ) by use of a system calibration factor derived from the imaging of a mouse-size water equivalent. Three-dimensional region-ofinterest analysis of reconstructed images (ASIPro software, Concorde Microsystems) yielded tumor and normal organ activity concentrations (i.e., mean, maximum, and $\mathrm{SD}$ of the \% $\mathrm{ID} / \mathrm{g}$ ). Tumor-to-muscle activity concentration ratios were derived by dividing image-derived tumor $\% \mathrm{ID} / \mathrm{g}$ values by $\gamma$-counter-derived muscle $\% \mathrm{ID} / \mathrm{g}$ values.

In vivo $\alpha_{v} \beta_{3}$ integrin receptor blocking studies. Average tumor uptake values of ${ }^{124}$ I-cRGDY-PEG-dots $(300 \mu \mathrm{Ci})$ were determined before and 30 minutes after i.v. injection of excess of cRGD peptide ( $6 \mu \mathrm{g} / \mathrm{ml}$ PBS). Initial uptake values were determined 3 days prior to blocking.

Nodal mapping and clearance of particles using multiscale fluorescence imaging techniques. Nude mice bearing hind leg tumors were injected by 4-quadrant, peritumoral administration of a 50- $\mu$ l PEG-dots or cRGDY-PEG-dot sample and allowed to perambulate freely. Mice were anesthetized with a $2 \%$ isoflurane/98\% oxygen mixture, and serial whole-body optical imaging of intact animals was performed at 10-, 20-, 30-, and 60-minutes p.i. and spectrally deconvolved. Several non-tumor-bearing mice were additionally injected with 50- $\mu$ l PEG-dots or PEG-cRGDY-PEG-dots subdermally in the hind leg. Superficial paramidline incisions were made along the ventral aspect of mice, surgically exposing the region from the hind limb to axilla, in order to enhance in situ optical detection of locoregional nodes (inguinal, axillary) and draining lymphatics, using both the whole-body optical scanner and a macroscopic fluorescence microscope fitted with $650 \pm 20 \mathrm{~nm}$ NIR excitation and 710-nm long-pass emission filters.

Imaging metastatic disease in spontaneous melanoma miniswine models. Spontaneous melanoma Sinclair miniature swine (10-12 kg, Sinclair Research Center) were injected i.v. with $5 \mathrm{mCi}{ }^{18} \mathrm{~F}-\mathrm{FDG}$ for dynamic whole-body PET imaging detection of nodal and/or organ metastases, followed by CT scan acquisition for anatomic colocalization. Additional dynamic PET-CT scans were acquired after subdermal, 4-quadrant peritumoral injections of ${ }^{124}$ I-RGD-PEG-dots to detect and localize metastatic disease and for presurgical planning. Imaged nodes were confirmed intraoperatively within the exposed surgical bed by visual inspection and $\gamma$-counting using hand-held PET devices prior to excision. Harvested specimens, as well as nodal basins bilaterally, were evaluated histologically with H\&E staining and were additionally stained with specific human melanoma markers (HMB45, PNL2 Dako, 1:100) according to standard protocols to confirm the presence of melanoma. Radioactivity levels were assessed within these tissue specimens for correlative purposes with the hand-held PET probe. Whole-body optical fluorescence imaging of excised specimens was additionally performed using the Maestro imaging system to identify nanoparticle fluorescence.

Statistics. Data are expressed as mean \pm SD and compared, where indicated, by 1-tailed Mann-Whitney $U$ test. We assigned statistical significance at $P<0.05$. Statistical analyses comparing groups of tumor mice receiving targeted/nontargeted probes or bearing M21/M21L tumors were performed and demonstrated statistical significance with $P<0.05$. For biodistribution studies, the tissue-specific mean $\% \mathrm{ID} / \mathrm{g}$ values of ${ }^{124} \mathrm{I}$-cRGDY-PEG-dots ( $n=7$ mice) and ${ }^{124} \mathrm{I}$-PEG-dots (control, $n=5$ mice) were compared at each time point, with statistically significant differences in tracer activities observed in blood, tumor, and major organs at 4- and 96-hours p.i. as well as at 24-hours p.i. for tumor and other tissues (Supplemental Table 1). For tumor targeting studies, differences in mean \% ID/g values between M21 $(n=7)$ and M21L tumor mice $(n=5)$, as well as mice receiving ${ }^{124} \mathrm{I}$-PEG-dots $(n=5)$ or ${ }^{124} \mathrm{I}$-cRADY-PEG$\operatorname{dot}(n=3)$ controls, were found to be maximal at 4-hours p.i. $(P=0.0015$ for PEG-dot and M21L controls; $P=0.01$ for cRADY-PEG-dot control), remaining significantly elevated at 24 hours $(P=0.0015$ and $P=0.004$, 
respectively), 48 hours ( $P=0.001$ and $P=0.003$, respectively), 72 hours $(P=0.015$ and $P=0.005$, respectively), and 96 hours $(P=0.005$ for M21M21L). Tumor-to-muscle ratios for ${ }^{124} \mathrm{I}$-cRGDY-PEG-dots $(n=7)$ versus those for ${ }^{124}$ I-PEG-dots $(n=5)$ were found to be statistically significant at 24 -hours p.i. $(P=0.001)$ and 72 -hours p.i. $(P=0.006)$ but not at 4-hours p.i. $(P=.35)$. Goodness-of-fit values $\left(\mathrm{R}^{2}\right)$, along with their associated $P$ values, were determined for the urine calibration curve $\left(\mathrm{R}^{2}=0.973\right.$, $P=0.01)$ as well as for the urine $\left(\mathrm{R}^{2}>0.95, P=0.047\right)$ and fecal $\left(\mathrm{R}^{2}>0.995, P<0.002\right)$ cumulative \% ID excretion curves using nonlinear regression analyses (SigmaPlot, Systat, v. 11.0).

\section{Acknowledgments}

We acknowledge M. Gönen for providing assistance with biostatistical analyses and R. Toledo-Crow, S. Patel, and J. Lewis for technical assistance. This work was supported by an NIH-American Recovery and Reinvestment Act/Clinical and Translational Science Center grant to M. Bradbury and U. Wiesner. The work was further supported by the Cornell Nanobiotechnology Center, a Science and
Technology Center program of the National Science Foundation under agreement no. ECS-9876771, and by the In vivo Cellular and Molecular Imaging Center P50 CA86438 grant. Technical services provided by the Memorial Sloan-Kettering Cancer Center SmallAnimal Imaging Core Facility, supported in part by NIH Small-Animal Imaging Research Program (SAIRP) grant no. R24 CA83084 and NIH center grant no. P30 CA08748, are gratefully acknowledged.

Received for publication March 23, 2011, and accepted in revised form May 4, 2011.

Address correspondence to: Michelle S. Bradbury, Department of Radiology, Sloan-Kettering Institute for Cancer Research, 1275 York Ave., New York, New York 10065, USA. Phone: 212.639.8938; Fax: 212.794.4010; E-mail: bradburm@mskcc.org.

Erik Herz's present address is: Exxon-Mobil Research and Engineering, Paulsboro, New Jersey, USA.
1. Weissleder R, Pittet MJ. Imaging in the era of molecular oncology. Nature. 2008;452(7187):580-589.

2. Weissleder R. Molecular imaging in cancer. Science. 2006;312(5777):1168-1171.

3. Manning HC, Lander A, McKinley E, Mutic NJ. Accelerating the development of novel molecular imaging probes: a role for high-throughput screening. J Nucl Med. 2008;49(9):1401-1404.

4. Fischer HC, Chan WC. Nanotoxicity: the growing need for in vivo study. Curr Opin Biotechnol. 2007;18(6):565-571.

5. Montet X, Funovics M, Montet-Abou K, Weissleder R, Josephson L. Multivalent effects of RGD peptides obtained by nanoparticle display. J Med Chem. 2006;49(20):6087-6093.

6. Montet X, Montet-Abou K, Reynolds F, Weissleder R, Josephson L. Nanoparticle imaging of integrins on tumor cells. Neoplasia. 2006;8(3):214-222.

7. Kelloff GJ, et al. The progress and promise of molecular imaging probes in oncologic drug development. Clin Cancer Res. 2005;11(22):7967-7985.

8. Heath JR, Davis ME. Nanotechnology and cancer. Annu Rev Med. 2008;59:251-265.

9. Hong H, Zhang Y, Sun J, Cai W. Molecular imaging and therapy of cancer with radiolabeled nanoparticles. Nano Today. 2009;4(5):399-413.

10. Burns AA, et al. Fluorescent silica nanoparticles with efficient urinary excretion for nanomedicine. Nano Lett. 2009;9(1):442-448.

11. Choi HS, et al. Design considerations for tumourtargeted nanoparticles. Nat Nanotechnol. 2010; $5(1): 42-47$.

12. Alric C, et al. Gadolinium chelate coated gold nanoparticles as contrast agents for both X-ray computed tomography and magnetic resonance imaging. J Am Chem Soc. 2008;130(18):5908-5915.

13. Lucignani G. Nanoparticles for concurrent multimodality imaging and therapy: the dawn of new theragnostic synergies. Eur J Nucl Med Mol Imaging. 2009;36(5):869-874.

14. Beer AJ, Schwaiger M. Imaging of integrin alphavbeta3 expression. Cancer Metastasis Rev. 2008; 27(4):631-644.

15. Rhyner M, Smith A, Gao X, Mao H, Yang L, Nie S. Quantum dots and targeted nanoparticles probes for in vivo tumor imaging. In: Bulte J, Modo M, eds. Nanoparticles in Biomedical Imaging. New York, New York, USA: Springer; 2008:413-426.

16. Hauck TS, Anderson RE, Fischer HC, Newbigging $\mathrm{S}$, Chan WC. In vivo quantum-dot toxicity assessment. Small. 2010;6(1):138-144.

17. Schipper ML, et al. Particle size, surface coating, and PEGylation influence the biodistribution of quantum dots in living mice. Small. 2009;5(1):126-134.
18. Zimmer JP, Kim SW, Ohnishi S, Tanaka E, Frangioni JV, Bawendi MG. Size series of small indium arsenide-zinc selenide core-shell nanocrystals and their application to in vivo imaging. J Am Chem Soc. 2006;128(8):2526-2527.

19. Aharoni A, Mokari T, Popov I, Banin U. Synthesis of InAs/CdSe/ZnSe core/shell1/shell2 structures with bright and stable near-infrared fluorescence. J Am Chem Soc. 2006;128(1):257-264.

20. Choi HS, et al. Renal clearance of quantum dots. Nat Biotechnol. 2007;25(10):1165-1170.

21. Ohnishi S, Lomnes SJ, Laurence RG, Gogbashian A, Mariani G, Frangioni JV. Organic alternatives to quantum dots for intraoperative near-infrared fluorescent sentinel lymph node mapping. Mol Imaging. 2005;4(3):172-181.

22. Kobayashi H, Brechbiel MW. Nano-sized MRI contrast agents with dendrimer cores. Adv Drug Deliv Rev. 2005;57(15):2271-2286.

23. Hilderbrand SA, Weissleder R. Near-infrared fluorescence: application to in vivo molecular imaging. Curr Opin Chem Biol. 2010;14(1):71-79.

24. Burns A, Ow H, Wiesner U. Fluorescent core-shell silica nanoparticles: towards "Lab on a Particle" architectures for nanobiotechnology. Chem Soc Rev. 2006;35(11):1028-1042.

25. Choi J, et al. Core-shell silica nanoparticles as fluorescent labels for nanomedicine. J Biomed Opt. 2007;12(6):064007.

26. Kumar R, et al. In vivo biodistribution and clearance studies using multimodal organically modified silica nanoparticles. ACS Nano. 2010;4(2):699-708.

27. Ow H, Larson DR, Srivastava M, Baird BA, Webb WW, Wiesner U. Bright and stable coreshell fluorescent silica nanoparticles. Nano Lett. 2005;5(1):113-117.

28. Kim S, et al. Near-infrared fluorescent type II quantum dots for sentinel lymph node mapping. Nat Biotechnol. 2004;22(1):93-97.

29. Tanaka E, Choi HS, Fujii H, Bawendi MG, Frangioni JV. Image-guided oncologic surgery using invisible light: completed pre-clinical development for sentinel lymph node mapping. Ann Surg Oncol. 2006;13(12):1671-1681.

30. Kobayashi $\mathrm{H}$, et al. Multimodal nanoprobes for radionuclide and five-color near-infrared optical lymphatic imaging. ACS Nano. 2007;1(4):258-264.

31. Felding-Habermann B, Mueller BM, Romerdahl $\mathrm{CA}$, Cheresh DA. Involvement of integrin alpha $\mathrm{V}$ gene expression in human melanoma tumorigenicity. J Clin Invest. 1992;89(6):2018-2022.

32. Ballou B, et al. Sentinel lymph node imaging using quantum dots in mouse tumor models. Bioconjug Chem. 2007;18(2):389-396.
33. Wang W, et al. Near-infrared optical imaging of integrin alphavbeta3 in human tumor xenografts. Mol Imaging. 2004;3(4):343-351.

34. Haubner R, Bruchertseifer F, Bock M, Kessler H, Schwaiger M, Wester HJ. Synthesis and biological evaluation of a (99m)Tc-labelled cyclic RGD peptide for imaging the alphavbeta 3 expression. Nuklearmedizin. 2004;43(1):26-32.

35. Larson DR, Ow H, Vishwasrao HD, Heikal AA, Wiesner U, Webb WW. Silica nanoparticle architecture determines radiative properties of encapsulated fluorophores. Chem Mater. 2008;20(8)2677-2684.

36. Cressman S, Sun Y, Maxwell E, Fang N, Chen D, Cullis P. Binding and uptake of RGD-containing ligands to cellular $\alpha_{v} \beta_{3}$ integrins. Int J Pept Res Ther. 2009;15(1):49-59.

37. Kimura RH, Cheng Z, Gambhir SS, Cochran JR. Engineered knottin peptides: a new class of agents for imaging integrin expression in living subjects. Cancer Res. 2009;69(6):2435-2442.

38. Li ZB, et al. (64)Cu-labeled tetrameric and octameric RGD peptides for small-animal PET of tumor alpha(v)beta(3) integrin expression. J Nucl Med. 2007;48(7):1162-1171.

39. Drickamer L. Rates of urine excretion by house mouse (mus domesticus): Differences by age, sex, social status, and reproductive condition. J Chem Eco. 1995;21(10):1481-1493.

40. Nahrendorf M, et al. Hybrid PET-optical imaging using targeted probes. Proc Natl Acad Sci U S A. 2010;107(17):7910-7915.

41. Seymour LW. Passive tumor targeting of soluble macromolecules and drug conjugates. Crit Rev Ther Drug Carrier Syst. 1992;9(2):135-187.

42. Haubner R, et al. Noninvasive imaging of alpha(v)beta3 integrin expression using 18F-labeled RGD-containing glycopeptide and positron emission tomography. Cancer Res. 2001;61(5):1781-1785.

43. Poethko T, et al. Two-step methodology for highyield routine radiohalogenation of peptides: (18)Flabeled RGD and octreotide analogs. J Nucl Med. 2004;45(5):892-902.

44. Cheng $Z$, et al. Small-animal PET of melanocortin 1 receptor expression using a $18 \mathrm{~F}-$-labeled alpha-melanocyte-stimulating hormone analog. J Nucl Med. 2007;48(6):987-994.

45. Bogush GH, Tracy MA, Zukoski Iv CF. Preparation of monodisperse silica particles: Control of size and mass fraction. J Non-Cryst Solids. 1988;104(1):95-106.

46. Sadasivan S, Dubey AK, Li Y, Rasmussen DH. Alcoholic solvent effect on silica synthesis-NMR and DLS investigation. J Sol-Gel Sci Technol. 1998; 12(1):5-14.

47. Herz E, Burns A, Bonner D, Wiesner U. Large 
stokes-shift fluorescent silica nanoparticles with enhanced emission over free dye for single excitation multiplexing. Macromol Rapid Commun. 2009;30(22):1907-1910.

48. Hermanson G. Bioconjugate Techniques. 2nd ed. London, United Kingdom: Academic Press; 2008.

49. Yoon TJ, et al. Specific targeting, cell sorting, and bioimaging with smart magnetic silica core-shell nanomaterials. Small. 2006;2(2):209-215.
50. Piatyszek MA, Jarmolowski A, Augustyniak J. IodoGen-mediated radioiodination of nucleic acids. Anal Biochem. 1988;172(2):356-359.

51. Eckerman K, Endo A. MIRD: Radionuclide Data and Decay Schemes. 2nd ed. Reston, Virginia, USA: Society of Nuclear Medicine; 2008.

52. Cristy M, Eckerman K. Specific absorbed fractions of energy at various ages from internal photon sources (I-VII). In: Oak Ridge National Laboratory Report. Spring- field, Virginia, USA: National Technical Information Service, Department of Commerce; 1987.

53. Loevinger R, Budinger T, Watson E. MIRD Primer for Absorbed Dose Calculations. Revised ed. New York, New York, USA: Society of Nuclear Medicine; 1991.

54. Stabin MG, Sparks RB, Crowe E. OLINDA/EXM: the second-generation personal computer software for internal dose assessment in nuclear medicine. J Nucl Med. 2005;46(6):1023-1027. 